\title{
Temperature Dependence of Collisional Deactivation of Highly Vibrationally Excited Biphenylene
}

\author{
By N. Fay and K. Luther*
}

Max-Planck-Institut für biophysikalische Chemie, Am Faßberg, D-37077 Göttingen and Institut für Physikalische Chemie der Universität Göttingen, Tammannstraße 6, D-37077 Göttingen, Germany

Dedicated to Prof. Dr. Dr. h.c. mult. H. Gg. Wagner on the occasion of his 70th birthday

(Received March 8, 2000)

\section{Energy Transfer / Vibrational Relaxation / Collision Dynamics / Gas Kinetics}

\begin{abstract}
Collisional energy transfer between highly vibrationally excited biphenylene and a variety of mono- and polyatomic bath gases has been measured at temperatures between 333 and $523 \mathrm{~K}$. Biphenylene molecules were initially prepared with an additional vibrational energy of $28490 \mathrm{~cm}^{-1}$ by electronic absorption at $351 \mathrm{~nm}$ followed by fast internal conversion. The collisional deactivation was observed by detection of the energy dependent UV absorption at the red edge of the strong band at $255 \mathrm{~nm}$. A carefully tested empirical energy calibration, based on high temperature shock tube data was used. A negative temperature dependence of $\langle\Delta \mathrm{E}\rangle$, referred to Lennard-Jones collision numbers $\mathrm{Z}_{\mathrm{LJ}}$, was found for the vibrational energies between 10000 and $30000 \mathrm{~cm}^{-1}$ in all rare gases, $\mathrm{N}_{2}$, $\mathrm{CO}_{2}, \mathrm{SF}_{6}$, ethane, butane and heptane as colliders. This temperature dependence is only very weak in light rare gases and increases systematically with the efficiency of the collider. The absolute values of $\langle\Delta \mathrm{E}\rangle$ are analyzed in comparison with recent data sets on related larger polyatomic molecules and with results of trajectory calculations. In the discussion of the measured temperature dependence the problems with a proper distinction between the T-dependence of $\langle\Delta \mathrm{E}\rangle$ and of the collision number are also addressed.
\end{abstract}

\section{Introduction}

Collisional energy transfer is often a key process in the dynamics of chemical reactions in which highly vibrationally excited molecules occur as inter-

* Author to whom correspondence should be addressed. E-mail: kluther@gwdg.de 
mediates, for instance after thermal, optical or chemical activation. This has always been a strong motivation for the special interest in vibrational relaxation at energies of chemical relevance which usually correspond to the regime of very high densities of molecular states. Deactivation of high degrees of vibrational excitation has been studied very frequently and the use of various laser techniques has led to an impressive progress in quality and detail of information [1]. Nevertheless it is fair to say that collisional energy transfer at high densities of states is not yet well understood and less than its reactive counterpart. As theories with sufficient predictive power for applications are also still missing it is even more important to extend the reliable basis of systematic empirical data on this type of molecular interaction.

Some aspects of vibrational deactivation with strong relevance for reaction dynamics have lately become much clearer, like the problem of pressure dependence of vibrational relaxation and its deviations from linear scaling behaviour at higher collider densities [2-4]. However in the field of temperature dependence of collisional energy transfer - obviously important in combustion and high temperature kinetics - the experimental results still provide a rather inhomogenous picture. Although more recently some consistent basis of reliable data appears to cristallize out still much more detailed material is needed in support.

It is the aim of this work to provide accurate data of vibrational relaxation and its temperature dependence for a new molecular example related to the group of aromatic compounds so far studied on a comparable level. This should provide increasing possibilities for the identification of parametric influences on relaxation rates and for conclusions on dynamical details.

Among the various methods developed to directly observe collisional deactivation of highly vibrationally excited molecules the detection of energy dependent UV-absorption (UVA) [5-8] or of IR-emission (IRF) [912] have been used most frequently. In both methods, as in comparable ones [13] the average amount of energy transferred per collision - the "first moment of energy transfer" - $\langle\Delta \mathrm{E}\rangle(\mathrm{E})$ can be determined, actually averaged over the varying molecular energy distribution during relaxation, i.e. as $\langle\langle\Delta \mathrm{E}\rangle\rangle$. From observation of time dependent light intensity in absorption or emission deactivation rates are measured. High quality data on $\langle\Delta \mathrm{E}\rangle(\mathrm{E})$ can rather easily be gathered, provided calibration curves of sufficient quality are available, like e.g. $\varepsilon(\mathrm{E}, \mathrm{T})$ in case of UVA. In contrast to the previous techniques the recent KCSI-method [14-16] is able to monitor the full evolution of relaxing molecular populations in its shape and displacement on the vibrational energy scale by selective ionization measurement at various narrow "energy windows". It can thus provide the complete information on energy transfer probabilities $\mathrm{P}\left(\mathrm{E}^{\prime}, \mathrm{E}\right)$, including first moments $\langle\Delta \mathrm{E}\rangle$ and higher ones $\left\langle\Delta \mathrm{E}^{\mathrm{n}}\right\rangle$. Its unequalled sensitivity and robustsness in the determi- 
nation of $\langle\Delta E\rangle(E)$ makes it a preferential choice for the determination of benchmark data. However because of technical and molecular constraints it is less widely applicable and versatile than the simpler former methods. Complementary types of information can be gathered under certain conditions when state selective detection is possible of the energy accepted by bath gas molecules in single collisions [17]. In special bath gases, mainly $\mathrm{CO}_{2}$, the change in rotational, vibrational and translational energy due to a collision can thus be determined in detail for certain energy ranges as well as parts of $\mathrm{P}\left(\mathrm{E}^{\prime}, \mathrm{E}\right)$ distributions for very high $\Delta \mathrm{E}$.

Inspection of the available directly determined data on $\langle\Delta \mathrm{E}\rangle(\mathrm{E})$ for larger molecules ( $\geq$ benzene) shows, that the energy dependence of the first moment is quite well approximated by a linear function, at least at energies between 10 and $30 \times 10^{3} \mathrm{~cm}^{-1}$. Such linearity in $\langle\Delta \mathrm{E}\rangle(\mathrm{E})$ is reflected in an exponential time dependence $\langle\mathrm{E}\rangle(\mathrm{t})$ of relaxation, which is also found within the accuracy of the present work. Slight curvatures in $\langle\Delta E\rangle(E)$ which become more important at even higher energies and the steeper decline of $\langle\Delta \mathrm{E}\rangle$ at low energies are further details which can be measured accurately by the KCSI-method. In practice their reliable determination is mostly beyond the realistic possibilities of the UVA technique and other "first moment methods". However this is not a disadvantage for the present aim of well established sets of data on the temperature dependence of $\langle\Delta \mathrm{E}\rangle$ in the mentioned range of vibrational energies. It turns out that a critically handled data evaluation within the constraints of exponential energy decay - accepting its very slightly approximate character - provides an excellent possibility to considerably improve the accuracy of $\langle\Delta \mathrm{E}\rangle$ data from UVA and thus to determine the quantitative variations upon changes of temperature and bathgas on a consistently improved basis.

A uniform tendency of decreasing $\langle\Delta \mathrm{E}\rangle$ with the increase of temperature from 333 to $523 \mathrm{~K}$ was observed in all bath gases studied. It varied from very slight dependencies for light atomic colliders to relatively strong effects in large molecular bath gases. Comparison of the experimental results with results of our recent trajectory calculations in very similar systems provide a great support for the interpretation of the observed trends. The unresolved problem of the correct choice of a collision number $\mathrm{Z}$ in energy transfer turns out to be of special importance in discussions of bath gas temperature dependence at comparisons with predictions of molecular collision models.

\section{Experimental}

Vibrationally excited biphenylene was prepared by UV-pumping with a $10 \mathrm{~ns}$ laser pulse into the electronically excited $S_{2}$ state and the following very fast internal conversion of the molecule. The $S_{1}$ and the $S_{2}$ state both 


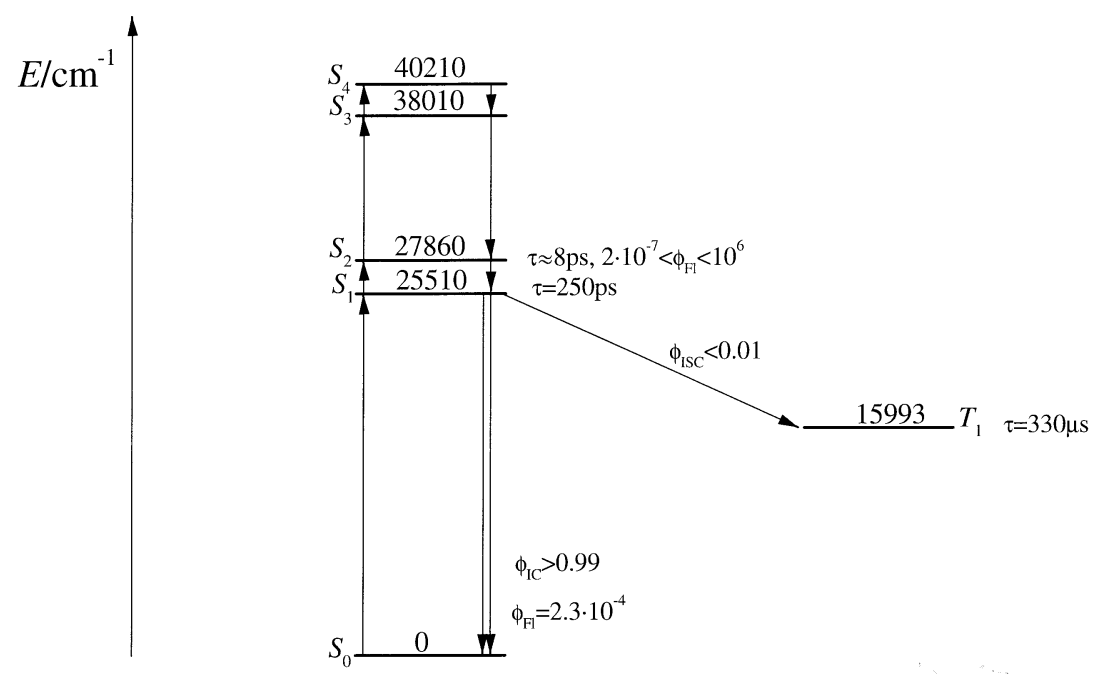

Fig. 1. Electronic energy level diagramm of biphenylene with lifetimes and quantum yields for intramolecular transitions.

have very short lifetimes ( $250 \mathrm{ps}$ and $8 \mathrm{ps}$ respectively) and the quantum yields of fluorescence $\left(\Phi_{\mathrm{F}}=2.3 \times 10^{-4}\right)$ and of intersystem crossing $\left(\mathrm{S}_{1} \rightarrow \mathrm{T}_{1}\right)\left(\Phi_{\mathrm{ISC}}<0.01\right)$ are correspondingly small such that more than $99 \%$ of the optically excited molecules undergo internal conversion into the $\mathrm{S}_{0}$ long before any collision occurs at the pressures of the experiments. The energy level diagramm of biphenylene is shown in Fig. 1. Collisional deactivation was observed by monitoring the changes in absorption at $255 \mathrm{~nm}$, in the red wing of the $S_{3}$ absorption band. Details of the spectrum and of $\varepsilon(\mathrm{E})$ are discussed further down (section 3.1).

The experimental set up was a modified version of earlier, proven arrangements. A stainless steel cylindrical reaction cell with $60 \mathrm{~cm}$ optical path length and $20 \mathrm{~mm}$ inner diameter was equipped with sapphire windows and copper sealing for evacuation to $5 \times 10^{-6} \mathrm{mbar}$. The windows were tilted by $10^{\circ}$ with respect to the optical axis in a position retracted inward from the faces of the cell body to minimize influences of convective cooling at the higher temperatures. Evenly distributed electrical heat tape allowed to keep the cell at temperatures up to $600 \mathrm{~K}$ stabilized within $1 \mathrm{~K}$. All parts close to the cell or in contact with it were made of stainless steel and heated as well, including a seperate reservoir for byphenylene with a direct, valve controlled connection to the cell. For low pressure measurements $<1$ mbar a capacitance manometer was used thermostabilized at $125^{\circ} \mathrm{C}$ to avoid condensation problems with biphenylene. After evacuation the cell was first filled with 5-10 $\mu$ bar biphenylene. Between 2 and 10 mbar collider gas 
were then added fast through a capillary valve at conditions of minimized backflux. The leak rate was $10^{-6} \mathrm{mbar}^{-1} \mathrm{~s}^{-1}$ at $\mathrm{T}=333$ and $413 \mathrm{~K}$, but higher at $523 \mathrm{~K}$. Slight systematic changes in pressure at this highest temperature during longer series of measurements were checked and found to be taken well into account by allowing for $<2 \%$ systematic error in the relaxation rates at $523 \mathrm{~K}$.

A XeF excimer laser was used for $10 \mathrm{~ns}$ pump pulses at $351 \mathrm{~nm}$. After expansion of the beam diameter by a telescope only a central part with very even intensity distribution was used inside the cell. A selective dielectric mirror on quartz under $45^{\circ}$ folded the laser beam into the optical cell axis in front of the cell and another such mirror removed it from the analyis beam right after the passage through the cell and send it on to a joulemeter which measured the energy of each pump pulse. Variations in the laser energy were below $5 \%$. Effective fluences of $3 \mathrm{~mJ} / \mathrm{cm}^{2}$ were used. With $\varepsilon=40001 \mathrm{~mol}^{-1} \mathrm{~cm}^{-1}$ at $351 \mathrm{~nm}$ the degree of excitation at the typical pressures of biphenylene remained at $6 \pm 1 \%$ along the length of the cell with a negligable contribution of two photon absorption smaller by about a factor of 30. After the excitation at $351 \mathrm{~nm}$ and internal conversion the molecules are prepared with an vibrational energy of $28490 \mathrm{~cm}^{-1}$ in addition to their thermal energy content.

The collimated analysis beam from a Xe-arc lamp passed the cell counterpropagating beam in beam with the pump pulse. Beam diameaters were set by apertures such that the analysis beam with its smaller diameter passed along the cell axis well inside the volume of excitation. The absorption profiles were recorded with a monochromator (Spex $270 \mathrm{M})$ at a resolution of $\Delta \lambda( \pm 10 \%)=0.6 \mathrm{~nm}$ and a photomultiplier (1P28A) connected to a digital oscilloscope (Tektronix TDS640). Calibration spectra $\varepsilon(\mathrm{E})$ of biphenylene were taken at temperatures between $370 \mathrm{~K}$ and $1600 \mathrm{~K}$ either with a heatable cell in a Cary 5E spectrometer or by means of shock tube experiments and a spectrograph with a spectrally dispersing streak camera at the higher temperatures. The wavelength $\lambda=255 \mathrm{mn}$ was chosen to monitor the profiles of vibrational energy loss versus time.

The large influence of seemingly small fractions of effective colliders on the measured relaxation is often underestimated and had to be considered as the cell contained unevitably most of its biphenylene (95\% in our case) unexcited as a second type of collider molecules. The validity of a "linear mixing rule" for mixtures of colliders with different efficiencies has been reported earlier and was recently tested experimentally in a very similar system to be a very good first order approximation [18]. On this basis a systematic deviation $\Delta$ is given by

$$
\Delta=(\mathrm{pZ}\langle\Delta \mathrm{E}\rangle)_{\mathrm{BPh}-\mathrm{BPh}} /(\mathrm{pZ}\langle\Delta \mathrm{E}\rangle)_{\mathrm{BPh}_{\mathrm{M}}} .
$$

The value of $\Delta$ was kept close to $1 \%$ which means that for inefficient atomic colliders $\mathrm{M}$ the ratio $[\mathrm{BPh}] /[\mathrm{M}]$ was chosen to be $<10^{-3}$. Another 
aspect to be considered is the possibility of diffusional losses of excited molecules during the detection time out of the observation volume. Diffusion was calculated by the standard expressions $\left\langle\Delta \mathrm{x}^{2}\right\rangle=2 \mathrm{Dt}$ and

$$
\mathrm{D}=(3 / 16 \pi)\left(\mathrm{p} \sigma_{\mathrm{LJ}}^{2} \Omega^{(1.1)^{\prime}}\right)^{-1}\left(2 \pi \mathrm{k}_{\mathrm{B}} \mathrm{T}^{3} / \mu\right)^{-1}
$$

with Lennard-Jones parameters $\sigma_{\mathrm{LJ}}$ and reduced collision integrals $\Omega^{(1.1)^{*}}$ from Ref. [19]; $\mu$ is the reduced mass. For a time interval of $5 \mu$ s e.g. a $\Delta \mathrm{x}=0.1 \mathrm{~mm}$ results in $8 \mathrm{mbar} \mathrm{Ar}$ at $333 \mathrm{~K}$. The observed relaxation times were kept below $5 \mu \mathrm{s}$. Additionally the observation volume was well enclosed within the larger diameter excitation volume, which results in an initial "plateautime" when only particle exchange but no effective diffusional losses occur in the detection zone. Any influence of diffusion could thus be excluded for all temperatures and gases of experiments.

The time resolution of the detection electronics was adjusted to provide high $\mathrm{S} / \mathrm{N}$ signal quality and from this point of view longer detection times would have been preferable. However for the reasons given above experimental conditions with relaxation within $5 \mu$ s were used. Instead the $\delta$-pulse response of our electronic circuit (100 ns fwhh) was carefully measured and the empirical response function was always used for convolution with the modelling curves throughout the data evaluation. Tests showed that under our conditions the results did not suffer in quality compared with those taken at a hypothetically unlimited time resolution. Averaging of up to 2000 pulses was used. At an energy input of $\leq 0.5 \mathrm{~mJ}$ per pulse additional bath gas heating was marginal on a single pulse basis and also as an cumulative effect. At a repetition rate of $10 \mathrm{~Hz}$ complete recooling took place between two pulses, a time span during which e.g. pracatically all gas molecules in the cell hit the metal walls at least once.

\section{Results}

\subsection{Absorption coefficients of hot biphenylene}

Electronic absorption spectra of molecules show pronounced dependences on the distributions of internal energy. In continuous bands this usually results in relatively simple dependences of the absorption coefficient $\varepsilon(\mathrm{E})$ on the content of rovibrational energy. It has been shown experimentally [20] and theoretically [21] that the spectra of microcanonical systems at energy $E^{\prime}$ are nearly the same as those of canoncial systems if $E^{\prime}$ is equal to the average $\langle\mathrm{E}\rangle(\mathrm{T})$ in the thermal systems. For larger molecules this is a very good approximation up to extremely high energies. Direct calculations of $\varepsilon(\mathrm{E})$ for larger molecules are mostly not available, and sufficiently accurate information on the potential surfaces often problematic. Semi-empirical parametrized models e.g. of Sulzer-Wieland [22] on the temperature depen- 


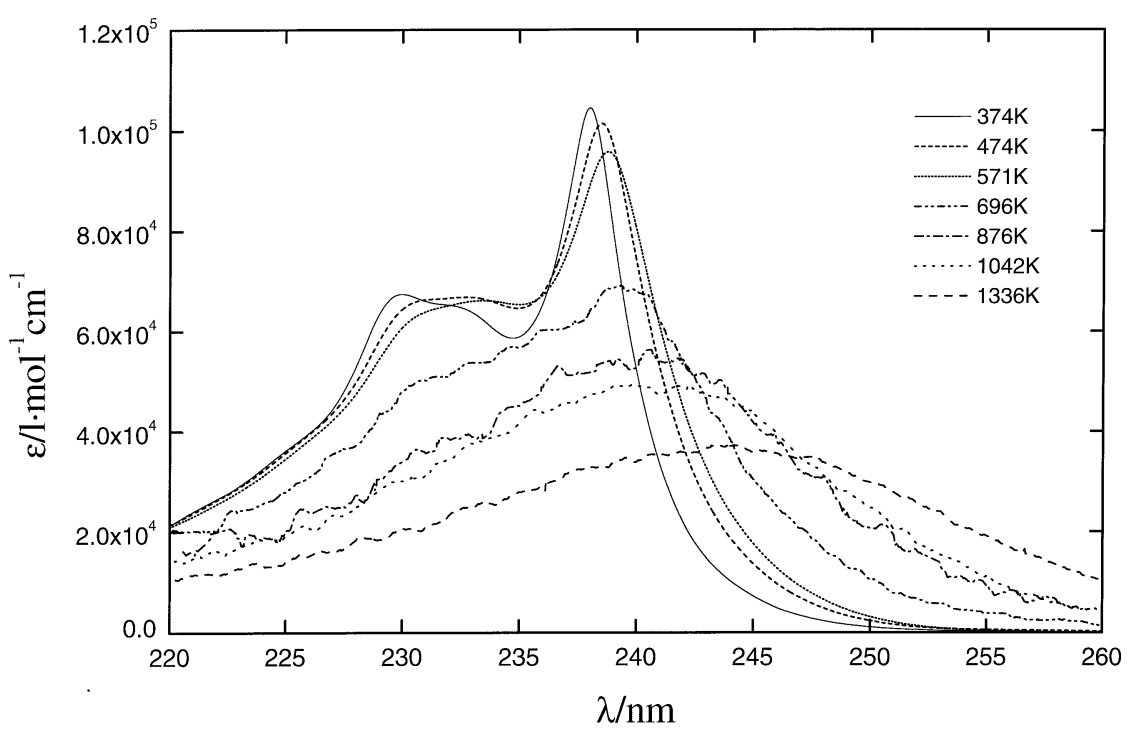

Fig. 2. Absorption spectra of hot biphenylene at various temperatures from 374 to $1336 \mathrm{~K}$.

dence of continous electronic absorption bands are very valuable for interpolation and limited extrapolation purposes but not sufficiently reliable if used alone. Thus in general good experimental calibration data from high temperature (e.g. shock tube) or laser excitation experiments are necessary to evaluate the $\varepsilon(\mathrm{E})$ dependence. Anyhow the calibration curve $\varepsilon(\mathrm{E})$ for vibrational relaxation measurements have to be prepared with great care as even seemingly small deviations can cause serious errors. (An example for dramatic effects of a harmless looking deviation from linearity in a calibration curve $\varepsilon(E)$ is shown in Ref. [16].) There are no simple rules to judge in advance how much various changes in the calibration will affect the modelling of observed data (see also the discussion below).

To determine our $\varepsilon(\langle\mathrm{E}\rangle)$ calibration curves of biphenylene, spectra were taken in a Cary $5 \mathrm{E}$ spectrometer at temperatures up to $540 \mathrm{~K}$ and in shock tube experiments up to $1600 \mathrm{~K}$. Fig. 2 shows examples of the biphenylene absorption spectra in the range of the $\mathrm{S}_{0} \rightarrow \mathrm{S}_{3} / \mathrm{S}_{0} \rightarrow \mathrm{S}_{4}$ transitions at various temperatures. The typical behaviour for an isolated band structure is seen with pronounced broadening and a redshift of the band maximum at rising temperature. $\lambda=255 \mathrm{~nm}$ was chosen as detection wavelength and series of additional shock tube measurements were made at $255 \mathrm{~nm}$ with a monochromator/photomulitplier arrangement for improved accuracy. To convert 


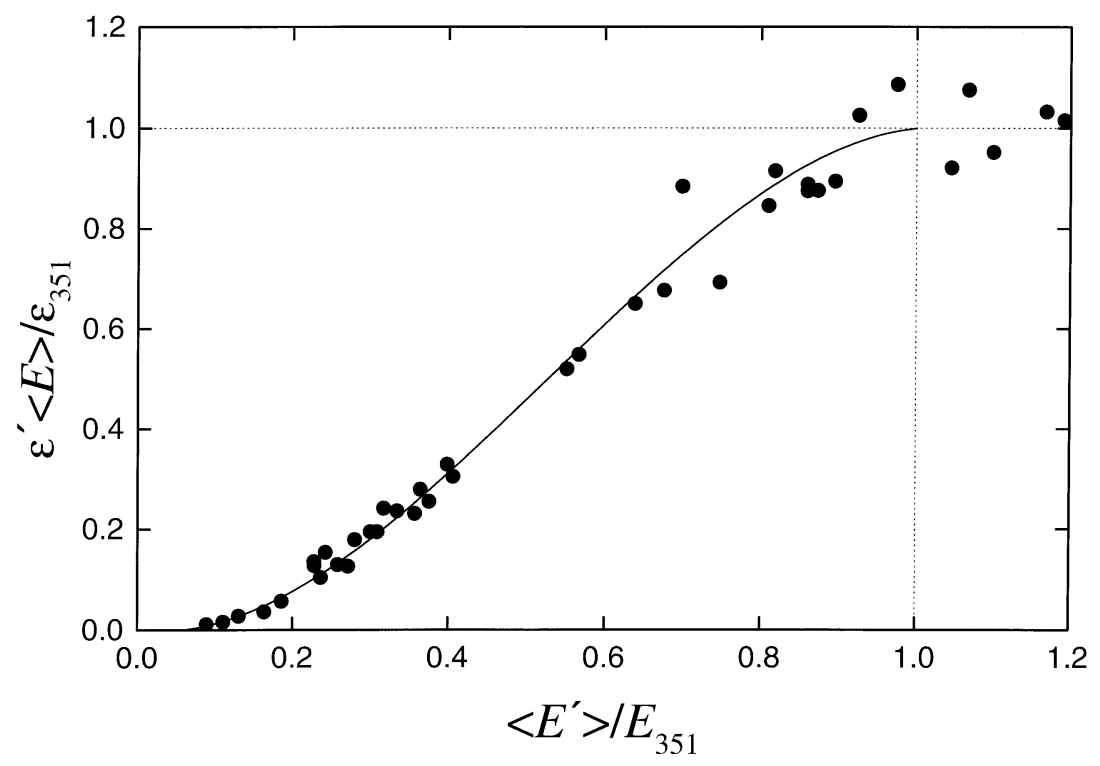

Fig. 3. Energy dependence of the biphenylene absorption coefficient $\varepsilon(255 \mathrm{~nm})$. Representation normalized for use at $\mathrm{T}=333 \mathrm{~K} .\left\langle\mathrm{E}^{\prime}\right\rangle=\langle\mathrm{E}\rangle-\langle\mathrm{E}\rangle_{\mathrm{T}}, \mathrm{E}_{351}=28490 \mathrm{~cm}^{-1}$ (excitation energy at $\lambda=351 \mathrm{~nm}), \varepsilon^{\prime}=\varepsilon(\langle\mathrm{E}\rangle)-\varepsilon\left(\langle\mathrm{E}\rangle_{\mathrm{T}}\right), \varepsilon_{351}=\varepsilon\left(\mathrm{E}_{351}+\langle\mathrm{E}\rangle_{\mathrm{T}}\right)-\varepsilon\left(\langle\mathrm{E}\rangle_{\mathrm{T}}\right)$. Points: data from static cell and shock tube measurements; full line: optimized fitting function (for the analytical expression at various $\mathrm{T}$ see text and Table 1).

$\varepsilon(\mathrm{T})$ into $\varepsilon(\mathrm{E})$ we calculated $\langle\mathrm{E}\rangle_{\mathrm{T}}=\langle\mathrm{E}\rangle(\mathrm{T})$ by means of $\langle\mathrm{E}\rangle=\sum_{\mathrm{i}=1}^{\mathrm{s}} \mathrm{h} v_{\mathrm{i}} /$ $\exp \left[\left(\mathrm{h} v_{\mathrm{i}} / \mathrm{k}_{\mathrm{B}} \mathrm{T}\right)-1\right]$ for the $\mathrm{s}=54$ vibrational modes of biphenylene. The frequencies and $\langle\mathrm{E}\rangle(\mathrm{T})$ are given in the Appendix. The resulting calibration points $\varepsilon(\mathrm{E})$ are shown in Fig. 3.

The measurements at $\mathrm{T}=333,413$ and $523 \mathrm{~K}\left(\langle\mathrm{E}\rangle_{\mathrm{BiPh}}=1530,2580\right.$ and $\left.4425 \mathrm{~cm}^{-1}\right)$ started after excitation at $\lambda=351 \mathrm{~nm}$ at rovibrational energies $\langle E\rangle_{0}=E_{351}+\langle E\rangle_{B i p h, T}$ of 30020,31070 and $32195 \mathrm{~cm}^{-1}$ respectively. To simplify the data evaluation reduced calibration curves were prepared with the total energy of deactivation $\mathrm{E}_{351}=28490 \mathrm{~cm}^{-1}$, normalized to a value of 1 and the 0 to 1 interval of relaxation shifted by the respective equilibrium bath energy $\langle\mathrm{E}\rangle_{\mathrm{T}}$. For further processing the monotonic normalized calibration curves were fitted within the 0 to 1 intervals by a third order polynomial

$$
\frac{\varepsilon^{\prime}(\langle\mathrm{E}\rangle)}{\varepsilon_{351}}=\kappa_{1}\left(\frac{\left\langle\mathrm{E}^{\prime}\right\rangle}{\mathrm{E}_{351}}\right)+\kappa_{2}\left(\frac{\left\langle\mathrm{E}^{\prime}\right\rangle}{\mathrm{E}_{351}}\right)^{2}+\kappa_{3}\left(\frac{\left\langle\mathrm{E}^{\prime}\right\rangle}{\mathrm{E}_{351}}\right)^{3}
$$

with $\left\langle\mathrm{E}^{\prime}\right\rangle=\langle\mathrm{E}\rangle-\langle\mathrm{E}\rangle_{\mathrm{T}}, \mathrm{E}_{351}=28490 \mathrm{~cm}^{-1}, \varepsilon^{\prime}(\langle\mathrm{E}\rangle)=\varepsilon(\langle\mathrm{E}\rangle)-\varepsilon\left(\langle\mathrm{E}\rangle_{\mathrm{T}}\right)$, and $\varepsilon_{351}=\varepsilon\left(\mathrm{E}_{351}+\langle\mathrm{E}\rangle_{\mathrm{T}}\right)-\varepsilon\left(\langle\mathrm{E}\rangle_{\mathrm{T}}\right)$. 
Table 1. Coefficients of the normalized calibration function $\varepsilon^{\prime}(\langle\mathrm{E}\rangle)$ at $\lambda=255 \mathrm{~nm}$ for relaxation between $\langle\mathrm{E}\rangle_{0}$ and $\langle\mathrm{E}\rangle_{\mathrm{T}}$ (see Eq. (1) and text).

\begin{tabular}{llllll}
\hline $\mathrm{T} / \mathrm{K}$ & \multicolumn{1}{l}{$\kappa_{1}$} & $\kappa_{2}$ & $\kappa_{3}$ & $\langle\mathrm{E}\rangle_{0}\left(\mathrm{~cm}^{-1}\right)$ & $\langle\mathrm{E}\rangle_{\mathrm{T}}\left(\mathrm{cm}^{-1}\right)$ \\
\hline 333 & -0.179 & 3.202 & -2.024 & 30020 & 1530 \\
413 & -0.048 & 2.952 & -1.905 & 31070 & 2580 \\
523 & 0.488 & 2.060 & -1.548 & 32915 & 4425 \\
\hline
\end{tabular}

The coefficients $\kappa_{\mathrm{n}}(\mathrm{T})$ are shown in Table 1. As an example the reduced calibration curve for measurements at $\mathrm{T}=333 \mathrm{~K}$ is shown in Fig. 3 .

\subsection{Modeling of $\varepsilon(t)$ profiles}

The evaluation of the experimental $\varepsilon(\mathrm{t})$ profiles starts from

$$
\frac{\mathrm{d}\langle\mathrm{E}(\mathrm{T})\rangle}{\mathrm{dt}}=[\mathrm{M}] \cdot \mathrm{Z}\langle\Delta \mathrm{E}(\mathrm{E})\rangle \text {. }
$$

With an energy independent collision number $\mathrm{Z}$ this is usually a very good approximation for narrow or specially shaped energy distributions still far from the final equilibrium. The inherent approximation $\langle\langle\Delta \mathrm{E}(\mathrm{E})\rangle\rangle=$ $\langle\Delta \mathrm{E}(\mathrm{E})\rangle$ which identifies the average $\Delta \mathrm{E}$ of the whole energy distribution of relaxing molecules with the $\langle\Delta \mathrm{E}(\mathrm{E})\rangle$ of a $\delta$-distribution at energy $\mathrm{E}$ cannot be avoided for all techniques which register at each instant the integral signals from the sum of all relaxing molecules.

The observed absorption-time profiles were modeled within the assumption of linear $\langle\Delta \mathrm{E}\rangle$ dependence on $\langle\mathrm{E}\rangle$ which corresponds to an exponential decay of energy in time:

$$
\langle\mathrm{E}(\mathrm{t})\rangle=\left\langle\mathrm{E}_{0}\right\rangle \exp \left(-\mathrm{t} / \tau_{\mathrm{c}}\right)
$$

with the relaxation rate constant $\mathrm{k}_{\mathrm{c}}=\tau_{\mathrm{c}}^{-1}$, the inverse of the relaxation time $\tau_{c}$, and the resulting energy dependence

$$
\langle\Delta \mathrm{E}(\mathrm{E})\rangle=-\langle\mathrm{E}\rangle\left(\mathrm{Z}_{\mathrm{LJ}} \cdot[\mathrm{M}] \cdot \tau_{\mathrm{c}}\right)^{-1} .
$$

This approximation is well justified by observations in more recent studies on molecules of comparable size in the range of $10-30 \times 10^{3} \mathrm{~cm}^{-1}$, where it was also used $[2,10,23]$. The validity is especially confirmed by the only small deviations from linearity at such energies determined by KCSI high precision measurements, which are especially sensitive in that respect $[15,16]$. If appropriate as in our case, the modeling within the boundary conditions of Eq. (3) has very positive consequences. The definiteness of the calibration curve through the experimental points can be controlled to a much higher precision than otherwise possible. To model directly the 


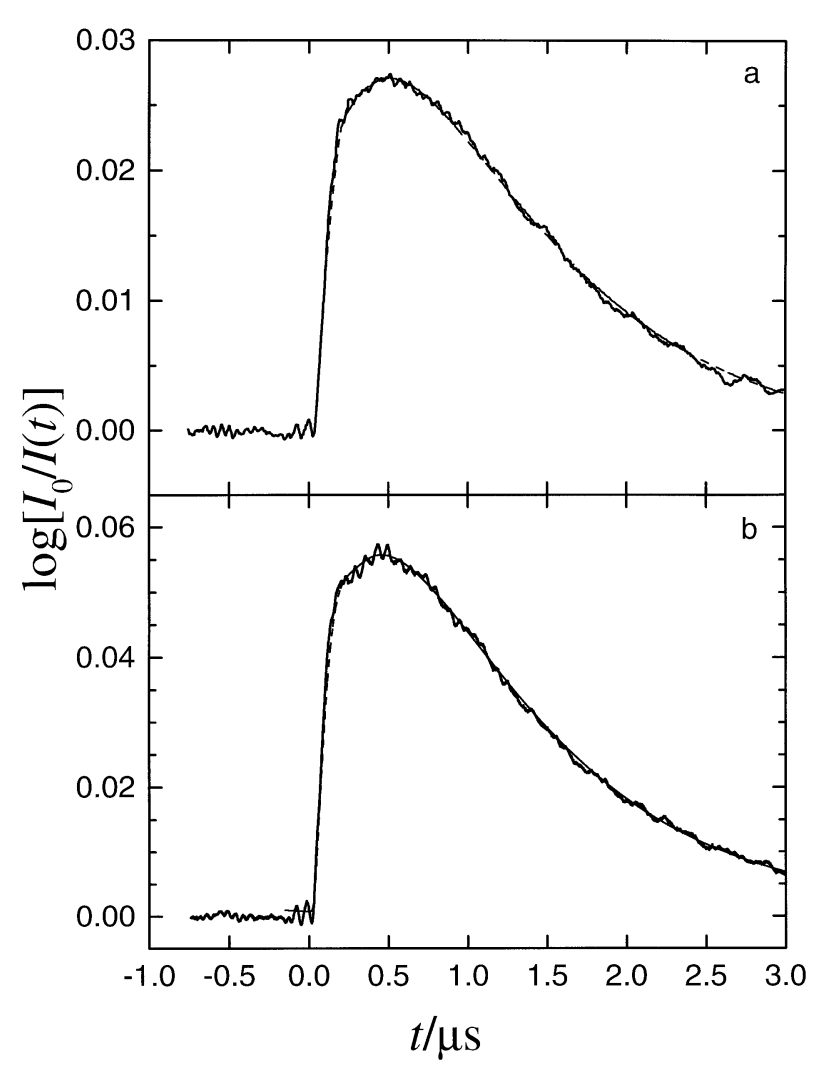

Fig. 4. Deactivation of biphenylene after vibrational excitation by $E_{351}=28490 \mathrm{~cm}^{-1}$ : a) 6.5 mbar neon, $\mathrm{T}=333 \mathrm{~K}$; b) 4.0 mbar $\mathrm{SF}_{6}, \mathrm{~T}=523 \mathrm{~K}$. Experimental absorptiontime profiles (full lines) and their simulations (dashed lines).

experimental $\log \left[\mathrm{I}_{0} / \mathrm{I}(\mathrm{t})\right]$ profiles the normalized calibration polynomials, Eq. (1), were convoluted with the measured detection circuit reponse function. Two examples for experimental absorption-time profiles and their simulations are shown in Fig. 4, the deactivation of biphenylene (a) in 6.5 mbar neon at $\mathrm{T}=333 \mathrm{~K}$ and (b) in $4.0 \mathrm{mbar}^{\mathrm{SF}_{6}}$ at $\mathrm{T}=523 \mathrm{~K}$.

The quality of fitting is much dependent on even small variations of the calibration curve. On the basis of the pure exponential $\langle E\rangle(t)$ behaviour, it is a severe consistency test if equally high fitting quality is reached for all measurements with various colliders and temperatures. With the calibration curves as given above very close to optimum exponential fits occured in all cases, within $\leq 5 \%$ in the resulting $\tau_{\mathrm{c}}$. Fig. 5 shows consequences of changes in shape and position of the calibration curve on the exponential fitting of the signal from Fig. $4 \mathrm{a}$. All three variations in Fig. 5a led to 

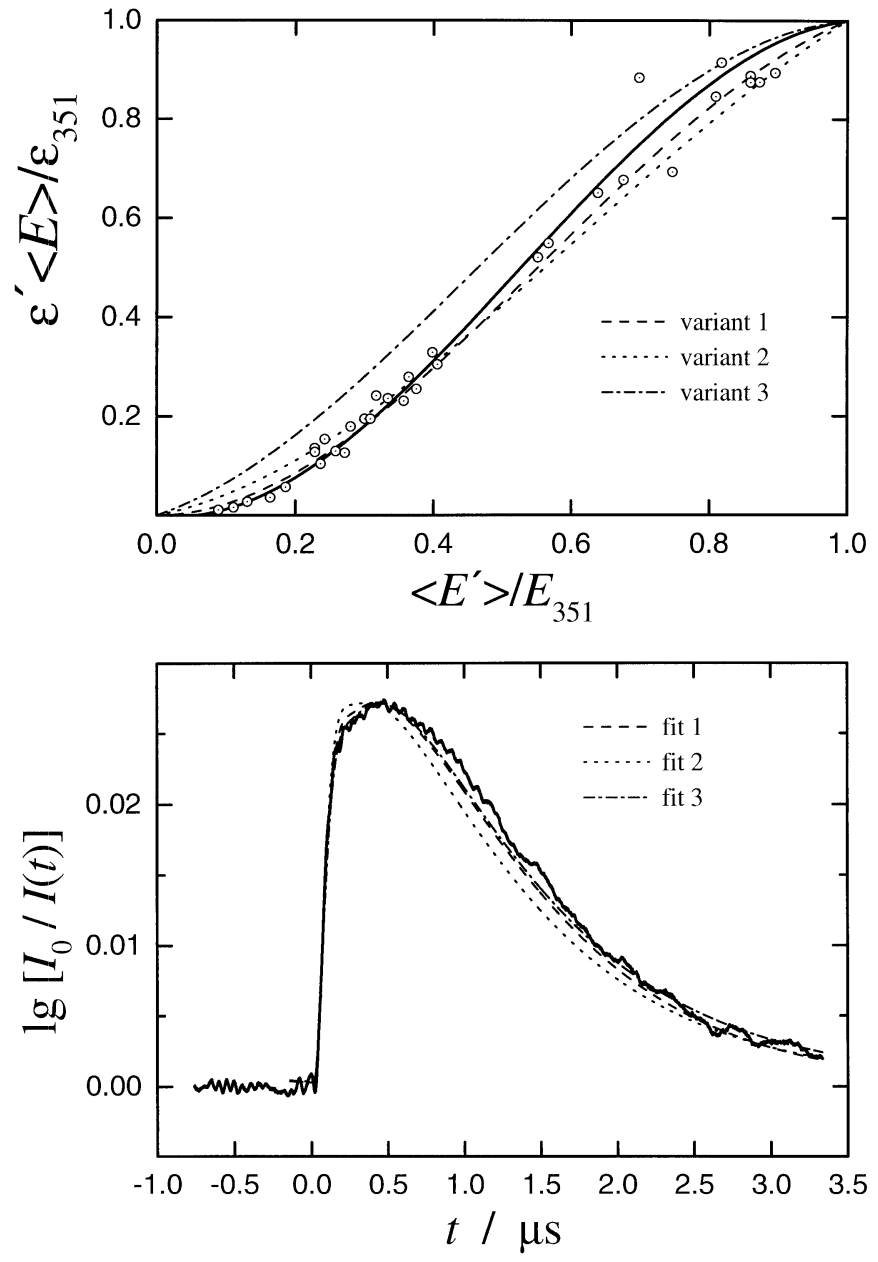

Fig. 5. Effects of various choices of the calibration curve $\varepsilon(\mathrm{E})$ on the simulation of experimental signals. Top: Points and full line are as in Fig. 3, the dashed and dotted variants 1-3 are examples for test calibration curves. Bottom: Signal from Fig. 4a. The curves 1-3 are the best exponential type simulations (as described in the text) based on the corresponding variants $1-3$ of calibration. The fit with the optimized calibration curve (full line) is seen in Fig. 4a.

clearly visible mismatching modeling curves in Fig. $5 \mathrm{~b}$ and illustrate the difficulties to judge the relative mismatch in advance. It is remarkable that variant 3, clearly far off from experimental points in Fig. 5a, can model the experimental curve in Fig. $5 \mathrm{~b}$ at least as good as variant 1 which is much closer to the optimum calibration curve. The choice of variant 2 , although 


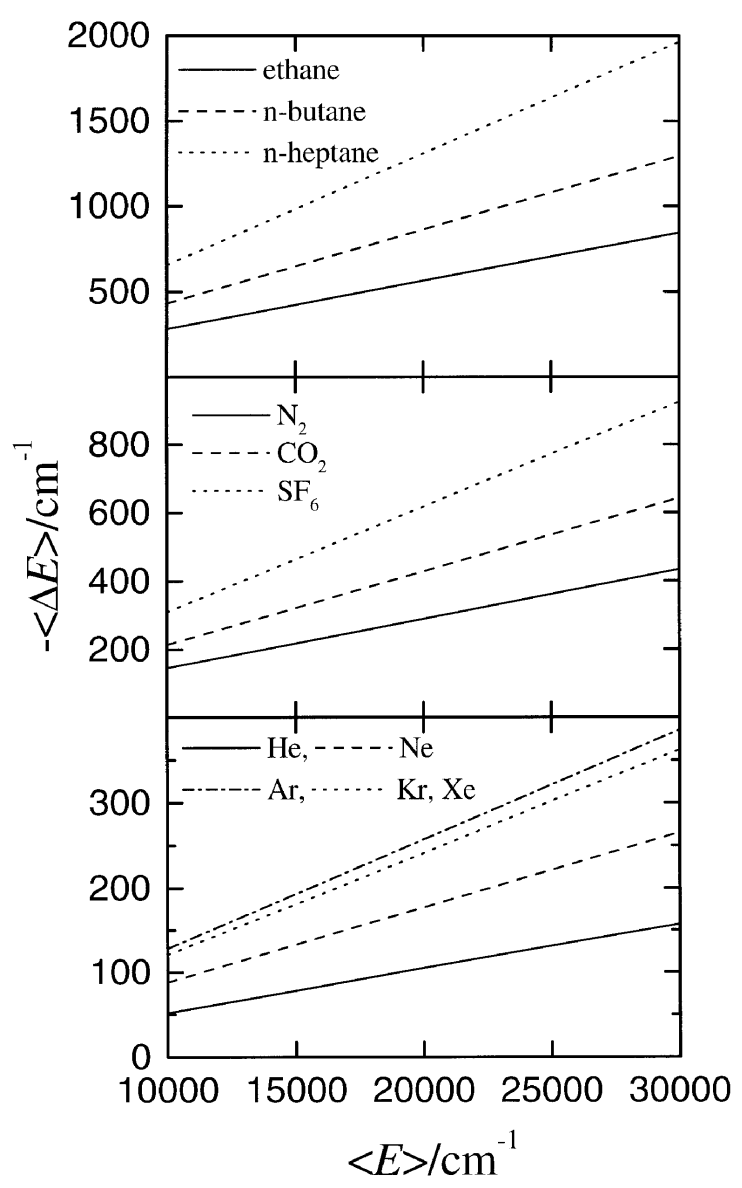

Fig. 6. Average energy transferred per collision $\langle\Delta \mathrm{E}\rangle$ as function of internal energy at $\mathrm{T}=333 \mathrm{~K}$ for various colliders (see also Table 3 ).

closer to the calibration points than variant 3 leads to a conspicious mismatch which could then e.g. be easily misinterpreted as indication of some apparent nonlinearity observed in $\langle\Delta \mathrm{E}(\mathrm{E})\rangle$. Such strong influence of even small trends in the calibration curve is mainly due to the fact that there is not a point to point correspondence between measured $\varepsilon(\mathrm{t})$ curves and the calibration function $\varepsilon(\mathrm{E})$. Instead each $\varepsilon(\mathrm{t})$ is related to some rather broad interval of the calibration curve weighted by the actual energy distribution.

The pure fitting errors of the simulation curves are very small due to the excellent signal to noise ratios. Deviation by $2 \%$ from optimum $\tau_{\mathrm{c}}$ are even recognized simply by eye. The overall accuracy of the $\Delta \mathrm{E}$ values is estimated to be within $\pm 10 \%$. 
Table 2. Lennard-Jones parameters of the bath gases and collision numbers $\mathrm{Z}_{\mathrm{LJ}}$ with biphenylene* at $\mathrm{T}=333 \mathrm{~K}, 413 \mathrm{~K}$, and $523 \mathrm{~K}$.

\begin{tabular}{|c|c|c|c|c|c|}
\hline \multirow[t]{2}{*}{ Collider } & \multirow{2}{*}{$\begin{array}{l}\sigma \\
\AA\end{array}$} & \multirow{2}{*}{$\begin{array}{l}\varepsilon / \mathrm{K}_{\mathrm{B}} \\
\mathrm{K}\end{array}$} & $\mathrm{Z}_{\mathrm{LJ}}^{333 \mathrm{~K}}$ & $\mathrm{Z}_{\mathrm{LJ}}^{413 \mathrm{~K}}$ & $\mathrm{Z}_{\mathrm{LJ}}^{523 \mathrm{~K}}$ \\
\hline & & & \multicolumn{3}{|c|}{$10^{7} \mathrm{mbar}^{-1} \mathrm{~s}^{-1}$} \\
\hline Helium & 2.55 & 10.2 & 1.820 & 1.568 & 1.339 \\
\hline Neon & 2.82 & 32 & 1.048 & 0.887 & 0.745 \\
\hline Argon & 3.47 & 114 & 1.144 & 0.938 & 0.765 \\
\hline Krypton & 3.66 & 178 & 1.024 & 0.829 & 0.667 \\
\hline Xenon & 4.05 & 230 & 1.041 & 0.838 & 0.669 \\
\hline $\mathrm{N}_{2}$ & 3.74 & 82 & 1.294 & 1.071 & 0.882 \\
\hline $\mathrm{O}_{2}$ & 3.47 & 107 & 1.228 & 1.009 & 0.825 \\
\hline $\mathrm{SF}_{6}$ & 5.20 & 212 & 1.219 & 0.984 & 0.787 \\
\hline $\mathrm{CO}_{2}$ & 3.94 & 201 & 1.386 & 1.119 & 0.897 \\
\hline Ethane & 4.44 & 216 & 1.796 & 1.448 & 1.159 \\
\hline n-Butane & 5.40 & 307 & 1.815 & 1.456 & 1.153 \\
\hline n-Heptane & 6.65 & 351 & 1.933 & 1.549 & 1.223 \\
\hline n-Octane & 7.02 & 359 & 1.984 & 1.589 & 1.254 \\
\hline
\end{tabular}

* $\sigma=6.61 \AA ; \varepsilon / \mathrm{k}_{\mathrm{B}}=523 \mathrm{~K}$.

As an independent additional check of the apparatus and the data processing for systematic errors, some completely analogous measurements on the relaxation of azulene were carried out in argon and heptane to compare with data from the experimentally very different and precise KCSI method. Apart from a somewhat larger statistical error in these measurements very good agreement with the corresponding results of Ref. [15, 24] was achieved.

\section{$3.3\langle\Delta \mathrm{E}\rangle$ values}

The results on $\langle\Delta \mathrm{E}\rangle$ for the various temepratures and bath gases are presented according to Eq. (4) based on Lennard-Jones collision numbers $Z_{\mathrm{LJ}}$ as it is customary in the field. $\mathrm{Z}_{\mathrm{LJ}}$ is given by

$$
\mathrm{Z}_{\mathrm{LJ}}=\mathrm{N}_{\mathrm{A}} \cdot \sigma_{\mathrm{AB}}^{2} \sqrt{\frac{8 \pi \mathrm{RT}}{\mu_{\mathrm{AB}}}} \Omega_{\mathrm{AB}}^{(2.2)^{*}}
$$

with $\sigma_{\mathrm{AB}}=\left(\sigma_{\mathrm{AA}}+\sigma_{\mathrm{BB}}\right) / 2$ and $\mu_{\mathrm{AB}}=\mathrm{M}_{\mathrm{A}} \cdot \mathrm{M}_{\mathrm{B}} /\left(\mathrm{M}_{\mathrm{A}}+\mathrm{M}_{\mathrm{B}}\right) . \varepsilon_{\mathrm{LJ}}$ and $\sigma_{\mathrm{LJ}}$ from Ref. [19] were used as given in Table 2, to keep the "normalization constant" $Z_{\mathrm{LJ}}$ the same as in earlier work for easy comparison of data. More recent and precise data for the rare gases [25] would slightly change some numerical values, without changing the picture. The $\Omega_{\mathrm{AB}}^{(2.2)^{*}}$ were taken from Ref. [26]. Fig. 6 shows the energy dependence $\langle\Delta \mathrm{E}(\mathrm{E})\rangle$ for the various bath gases at $\mathrm{T}=333 \mathrm{~K}$ as an example. Values of $\langle\Delta \mathrm{E}\rangle$ for the bath gases at temperatures $\mathrm{T}=333,413$ and $523 \mathrm{~K}$ are given in Table 3 for some selec- 
Table 3. Energy and temperature dependent $\langle\Delta \mathrm{E}\rangle$ of the biphenylene deactivation.

\begin{tabular}{|c|c|c|c|c|c|c|c|c|c|}
\hline \multirow[b]{3}{*}{$\mathrm{T} / \mathrm{K}$} & \multicolumn{9}{|c|}{$-\langle\Delta \mathrm{E}\rangle\left(\mathrm{cm}^{-1}\right)$} \\
\hline & \multicolumn{3}{|c|}{$\langle E\rangle=10000 \mathrm{~cm}^{-1}$} & \multicolumn{3}{|c|}{$\langle E\rangle=20000 \mathrm{~cm}^{-1}$} & \multicolumn{3}{|c|}{$\langle E\rangle=30000 \mathrm{~cm}^{-1}$} \\
\hline & 333 & 413 & 523 & 333 & 413 & 523 & 333 & 413 & 523 \\
\hline Helium & 52 & 51 & 49 & 105 & 101 & 98 & 157 & 152 & 147 \\
\hline Neon & 88 & 86 & 86 & 177 & 172 & 171 & 265 & 258 & 257 \\
\hline Argon & 128 & 119 & 112 & 257 & 238 & 224 & 385 & 357 & 335 \\
\hline Krypton & 121 & 117 & 110 & 241 & 233 & 220 & 362 & 350 & 330 \\
\hline Xenon & 121 & 108 & 102 & 242 & 217 & 203 & 363 & 325 & 304 \\
\hline $\mathrm{N}_{2}$ & 145 & 133 & 125 & 289 & 267 & 250 & 434 & 400 & 375 \\
\hline $\mathrm{O}_{2}$ & 153 & - & - & 307 & - & - & 460 & - & - \\
\hline $\mathrm{CO}_{2}$ & 214 & 198 & 167 & 428 & 395 & 333 & 642 & 593 & 500 \\
\hline $\mathrm{SF}_{6}$ & 309 & 294 & 260 & 617 & 588 & 519 & 926 & 882 & 779 \\
\hline Ethane & 281 & 261 & 226 & 563 & 521 & 451 & 844 & 782 & 677 \\
\hline n-Butane & 431 & 397 & 323 & 862 & 794 & 646 & 1293 & 1191 & 969 \\
\hline n-Heptane & 655 & 554 & 458 & 1309 & 1108 & 916 & 1964 & 1662 & 1374 \\
\hline n-Octane & 781 & - & - & 1563 & - & - & 2345 & - & - \\
\hline
\end{tabular}

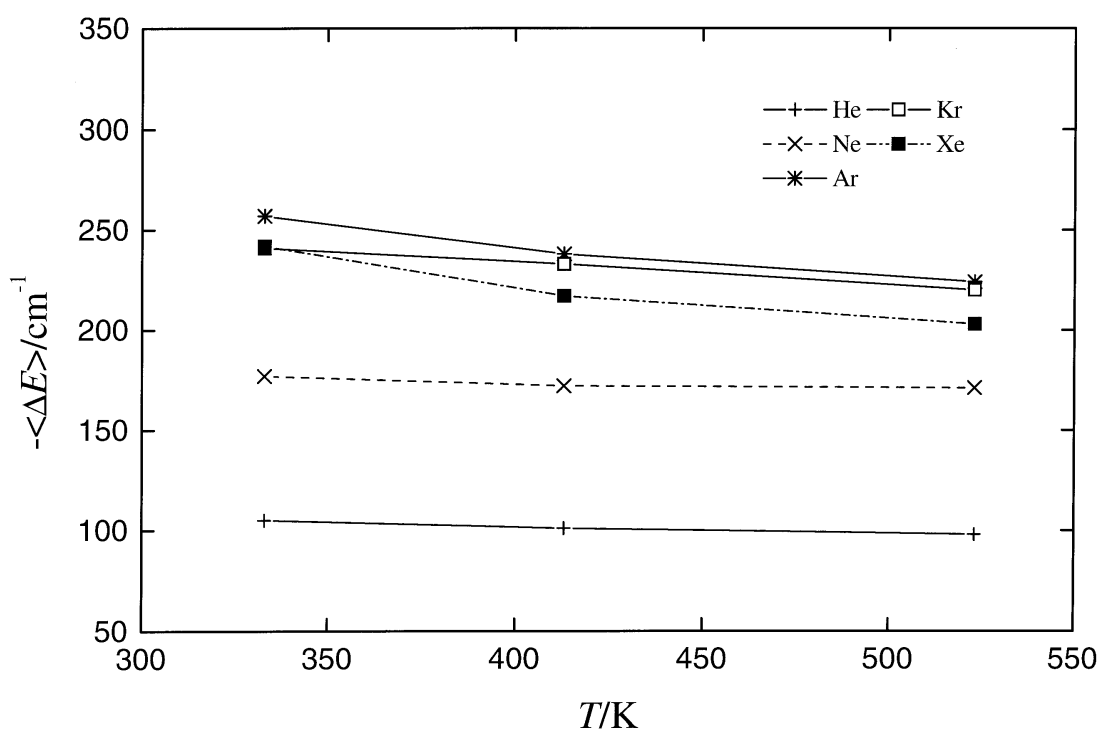

Fig. 7. Temperature dependence of $\langle\Delta E\rangle$ at internal energy $\langle E\rangle=20000 \mathrm{~cm}^{-1}$ for the colliders $\mathrm{He}, \mathrm{Ne}, \mathrm{Ar}, \mathrm{Kr}$ and $\mathrm{Xe}$ (see also Table 3 ). 


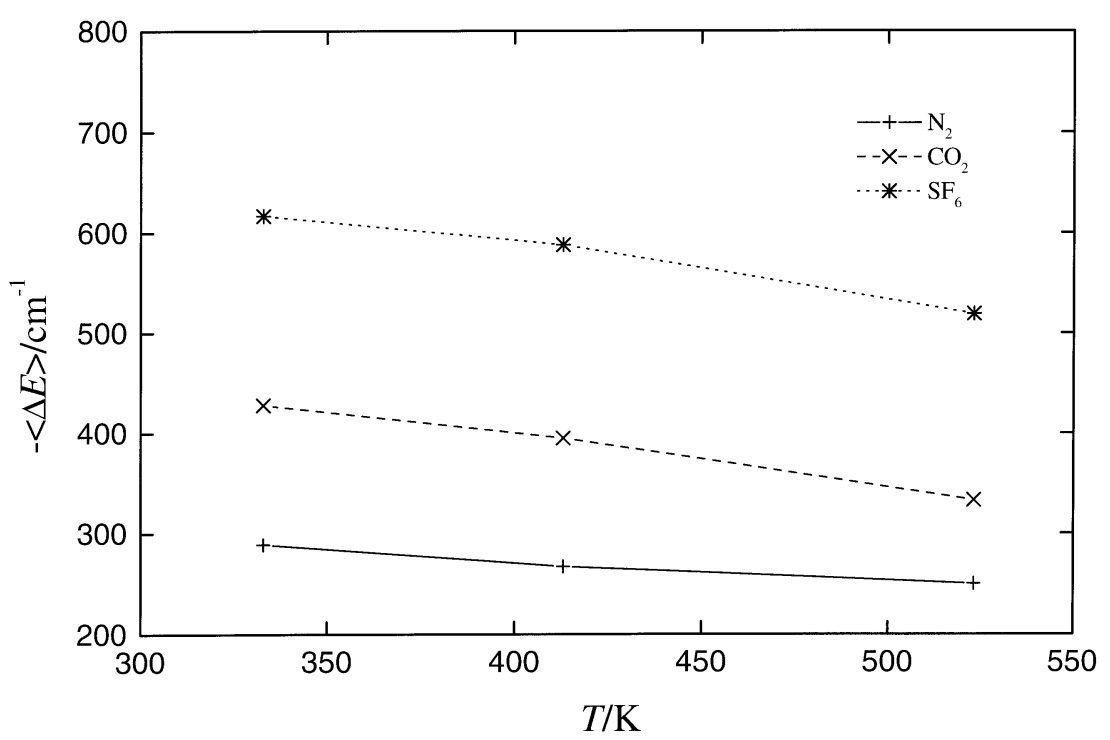

Fig. 8. As Fig. 7, for the colliders $\mathrm{N}_{2}, \mathrm{CO}_{2}$ and $\mathrm{SF}_{6}$.

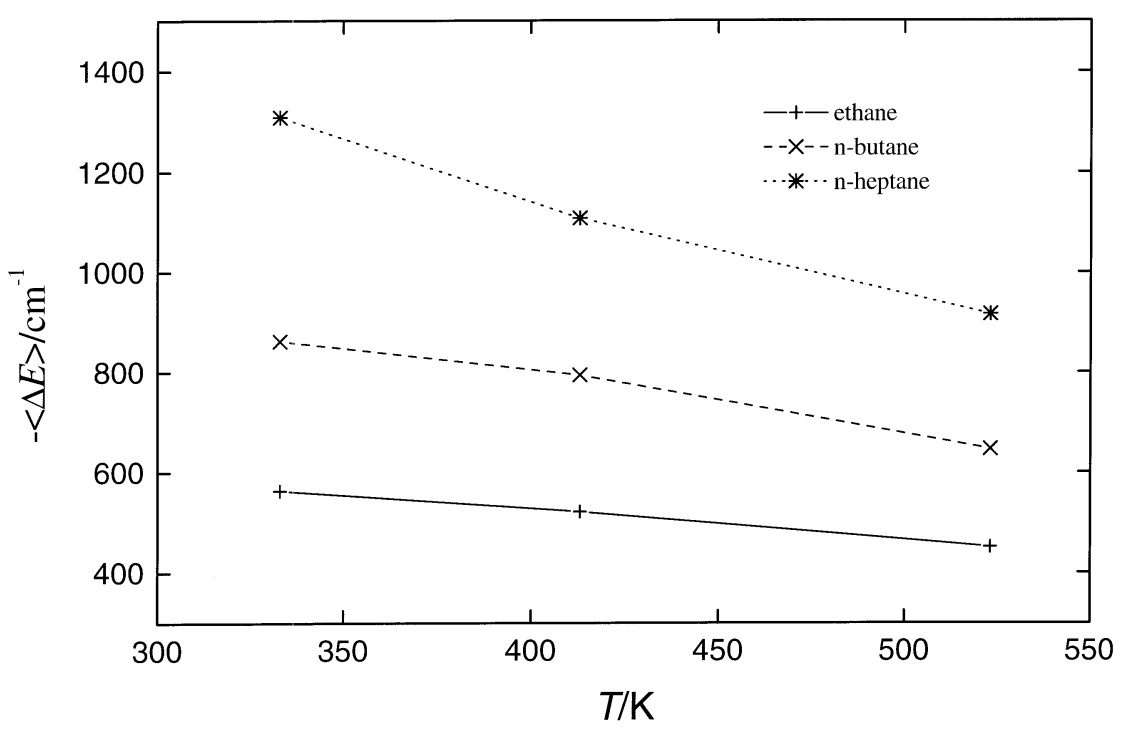

Fig. 9. As Fig. 7, for the colliders ethane, n-butane, and n-heptane. 
Table 4. Relaxation rate constants and coefficients of average energy transfer for deactivation of biphenylene at vibrational energies between 10 and $30 \times 10^{3} \mathrm{~cm}^{-1}$.

\begin{tabular}{|c|c|c|c|c|c|c|}
\hline \multirow[b]{2}{*}{$\mathrm{T} / \mathrm{K}$} & \multicolumn{3}{|c|}{$\mathrm{k}_{\mathrm{c}}[\mathrm{M}]^{-1} / 10^{-12} \mathrm{~cm}^{3} \mathrm{~s}^{-1}$} & \multicolumn{3}{|c|}{$\frac{-\langle\Delta \mathrm{E}\rangle \cdot 100^{*}}{\langle\mathrm{E}\rangle}$} \\
\hline & 333 & 413 & 523 & 333 & 413 & 523 \\
\hline Helium & 4.39 & 4.52 & 4.84 & 0.525 & 0.505 & 0.490 \\
\hline Neon & 4.25 & 4.35 & 4.57 & 0.883 & 0.860 & 0.850 \\
\hline Argon & 6.73 & 6.36 & 6.30 & 1.28 & 1.19 & 1.12 \\
\hline Krypton & 5.67 & 5.53 & 5.39 & 1.21 & 1.17 & 1.10 \\
\hline Xenon & 5.79 & 5.18 & 5.00 & 1.21 & 1.09 & 1.02 \\
\hline $\mathrm{N}_{2}$ & 8.61 & 8.15 & 8.12 & 1.45 & 1.34 & 1.25 \\
\hline $\mathrm{O}_{2}$ & 8.64 & - & - & 1.53 & - & - \\
\hline $\mathrm{CO}_{2}$ & 13.7 & 12.6 & 11.0 & 2.15 & 1.98 & 1.67 \\
\hline $\mathrm{SF}_{6}$ & 17.3 & 16.5 & 15.2 & 3.08 & 2.94 & 2.60 \\
\hline Ethane & 23.3 & 21.5 & 19.2 & 2.82 & 2.61 & 2.26 \\
\hline n-Butane & 38.4 & 32.9 & 27.4 & 4.32 & 3.97 & 3.23 \\
\hline n-Heptane & 58.2 & 48.9 & 40.4 & 6.55 & 5.55 & 4.58 \\
\hline n-Octane & 71.3 & - & - & 7.82 & - & - \\
\hline
\end{tabular}

$*-\langle\Delta \mathrm{E}\rangle /\langle\mathrm{E}\rangle=\mathrm{k}_{\mathrm{c}} /[\mathrm{M}] \cdot \mathrm{Z}_{\mathrm{LJ}}$ (see Eq. (4)).

ted energies. Figs. 7-9 show the results on the bath temperature dependence of $\langle\Delta \mathrm{E}\rangle(\mathrm{T})$ for the various colliders at a representative energy of $\langle E\rangle=20000 \mathrm{~cm}^{-1}$. Table 4 summarizes the results and shows a list of the reduced relaxation rate constants $\mathrm{k}_{\mathrm{c}} /[\mathrm{M}]$ and of the coefficients of average energy transferred, $\langle\Delta \mathrm{E}\rangle /\langle\mathrm{E}\rangle$, for all temperature and bath gas conditions of this work.

\section{Discussion}

\subsection{Bath gas and energy dependence of $\langle\Delta E\rangle$}

The various colliders of biphenylene show their same characteristic differences in relaxation efficiency at all temperatures and vibrational energies in this work. As exptected, the relative order in $\langle\Delta \mathrm{E}\rangle$ from the rare gases to larger polyatomics like heptane was found to be very similar to those reported for other larger molecules like toluene $[5,10,16]$ or azulene $[8,10$, 15]. A result which improves the possibilities to identify "normal" strongly collider specific properties in the energy transfer. The data in Table 3 quantify this with $\langle\Delta \mathrm{E}\rangle$ values at three vibrational energies $\langle\mathrm{E}\rangle(10,20$ and $30 \times 10^{3} \mathrm{~cm}^{-1}$ ) for $\mathrm{T}=333,413$ and $523 \mathrm{~K}$. An example of the energy dependences $\langle\Delta \mathrm{E}\rangle(\mathrm{E})$ for the various colliders is given in Fig. 6 for $\mathrm{T}=$ $333 \mathrm{~K}$. The variation of $\langle\Delta \mathrm{E}\rangle$ by a factor of about 12 between helium and heptane as bath gases at $333 \mathrm{~K}$ is only somewhat reduced to a factor of 9.4 at $523 \mathrm{~K}$. The increase of $\langle\Delta \mathrm{E}\rangle$ from He to the heavy rare gases with $\langle\Delta \mathrm{E}\rangle$ 
values close to that of a typical diatomic collider like $\mathrm{N}_{2}$ also confirm the "normal" order, without a special efficiency of $\mathrm{He}$ as bath gas. Only a relative high efficiency of argon is remarkable with $\langle\Delta \mathrm{E}\rangle 5-10 \%$ larger than in $\mathrm{Kr}$ or Xe. Slightly less pronounced, this effect has also been found for other cyclic compounds (toluene, azulene). It is supposed to be connected with the favourable position argon in particular can reach closely opposite of six membered ring structures and the resulting possibilities of increased interaction and energy transfer.

The question of strict $\langle\Delta E\rangle$ to $\langle E\rangle$ proportionality as seen in Fig. 6 shall be shortly readdressed. It has been explained above why this approximation was accepted for the data evaluation as a price for much improved accuracy and consistency of the attainable results. The nature and extent of minor systematic errors thus introduced can be well judged by comparison with the highly precise KCSI results in analogous cases: Slight but well resolved upward curvature towards high $\langle\mathrm{E}\rangle$ is generally found and a much stronger energy dependence in a narrow low energy range, which leads to an intercept at the ordinate if the closely linear part of $\langle\Delta E\rangle(E)$ is extrapolated. This intercept is extremely small for atomic colliders but not negligable for larger colliders at low energy. At $\langle E\rangle$ above $10000 \mathrm{~cm}^{-1}$ the influence remains very small, but in principle a tendency results that the slopes of $\langle\Delta \mathrm{E}\rangle$ derived under the assumption of strict exponential decay may be very slightly too steep for large colliders.

\subsection{Comparison with relaxation of other large molecules}

For a number of larger cyclic molecules there are now $\langle\Delta \mathrm{E}\rangle$ data available from direct KCSI, UVA or IRF measurements which are suitable for close comparison with respect to absolute values and energy dependence of $\langle\Delta \mathrm{E}\rangle(\mathrm{E})$. Table 5 shows representative values of $\langle\Delta \mathrm{E}\rangle$ at three vibrational energies $\langle\mathrm{E}\rangle\left(10,20\right.$ and $\left.30 \times 10^{3} \mathrm{~cm}^{-1}\right)$ at room temperature for $\mathrm{He}, \mathrm{Ar}, \mathrm{Xe}$ as atomic gases and $\mathrm{N}_{2}, \mathrm{CO}_{2}$, heptane as polyatomic colliders. For better comparison the biphenylene data are extrapolated from $333 \mathrm{~K}$ to $300 \mathrm{~K}$ according to our measured temperature dependence.

The similarities of $\langle\Delta \mathrm{E}\rangle$ for pyridine, pyrazine, toluene and even azulene are obvious, with a small systematic increase of the order of $10 \%$ in the cases of the heavier atomic colliders (Ar, Xe), which becomes better visible at the higher energies $\langle E\rangle$. While this could well be rationalized even within a simple Landau-Teller model of collisions, markedly larger $\langle\Delta \mathrm{E}\rangle$ are found in biphenylene. At the same time the relative increase in size or mass is not remarkable, when compared with the differences in mass or size e.g. between pyridine and azulene. While $\langle\Delta \mathrm{E}\rangle(\mathrm{He})$ is around $45 \%$ larger for the deactivation of biphenylene than for the other donor molecules in Table 5 this ratio is $>100 \%$ when relaxations in argon and xenon or similarly in nitrogen are compared. 
Table 5. Average energies transferred per collision from some larger polyatomic molecules at selected vibrational energies and $\mathrm{T}=300 \mathrm{~K} . \mathrm{M}=\operatorname{molar}$ mass $\left(\mathrm{g} \cdot \mathrm{mol}^{-1}\right)$.

\begin{tabular}{|c|c|c|c|c|c|c|}
\hline & & \multicolumn{5}{|c|}{$\langle\Delta \mathrm{E}\rangle(\mathrm{E})\left(\mathrm{cm}^{-1}\right)$} \\
\hline & & Pyridine $^{\mathrm{a}}$ & Pyrazine $^{\mathrm{a}}$ & Toluene $^{\mathrm{b}}$ & Azulene $^{\mathrm{c}}$ & Biphenylene $^{\mathrm{d}}$ \\
\hline Collider & $\langle\mathrm{E}\rangle\left(\mathrm{cm}^{-1}\right)$ & $\mathrm{M}=82$ & 82 & 92 & 128 & 152 \\
\hline Helium & $\begin{array}{l}1 \times 10^{4} \\
2 \times 10^{4} \\
3 \times 10^{4}\end{array}$ & $\begin{array}{r}40 \\
81 \\
127\end{array}$ & $\begin{array}{r}37 \\
72 \\
111\end{array}$ & $\begin{array}{r}38 \\
74 \\
114\end{array}$ & $\begin{array}{r}36 \\
72 \\
112\end{array}$ & $\begin{array}{r}53 \\
107 \\
159\end{array}$ \\
\hline Argon & $\begin{array}{l}1 \times 10^{4+} \\
2 \times 10^{4} \\
3 \times 10^{4}\end{array}$ & $\begin{array}{r}63 \\
123 \\
187\end{array}$ & $\begin{array}{r}63 \\
120 \\
180\end{array}$ & $\begin{array}{r}64 \\
125 \\
191\end{array}$ & $\begin{array}{r}68 \\
143 \\
225\end{array}$ & $\begin{array}{l}132 \\
266 \\
399\end{array}$ \\
\hline Xenon & $\begin{array}{l}1 \times 10^{4} \\
2 \times 10^{4} \\
3 \times 10^{4}\end{array}$ & $\begin{array}{r}69 \\
138 \\
213\end{array}$ & $\begin{array}{l}- \\
- \\
-\end{array}$ & $\begin{array}{r}68 \\
149 \\
239\end{array}$ & $\begin{array}{r}65 \\
145 \\
233\end{array}$ & $\begin{array}{l}127 \\
254 \\
382\end{array}$ \\
\hline $\mathrm{N}_{2}$ & $\begin{array}{l}1 \times 10^{4} \\
2 \times 10^{4} \\
3 \times 10^{4}\end{array}$ & $\begin{array}{r}87 \\
151 \\
219\end{array}$ & $\begin{array}{r}76 \\
133 \\
193\end{array}$ & $\begin{array}{l}- \\
- \\
-\end{array}$ & $\begin{array}{l}- \\
- \\
-\end{array}$ & $\begin{array}{l}151 \\
300 \\
451\end{array}$ \\
\hline $\mathrm{CO}_{2}$ & $\begin{array}{l}1 \times 10^{4} \\
2 \times 10^{4} \\
3 \times 10^{4}\end{array}$ & $\begin{array}{l}128 \\
237 \\
345\end{array}$ & $\begin{array}{l}123 \\
215 \\
299\end{array}$ & $\begin{array}{l}129 \\
271 \\
422\end{array}$ & $\begin{array}{l}154 \\
264 \\
372\end{array}$ & $\begin{array}{l}222 \\
445 \\
666\end{array}$ \\
\hline Heptane & $\begin{array}{l}1 \times 10^{4} \\
2 \times 10^{4} \\
3 \times 10^{4}\end{array}$ & $\begin{array}{r}497 \\
766 \\
1116\end{array}$ & $\begin{array}{r}523 \\
983 \\
1160\end{array}$ & $\begin{array}{r}633 \\
1160 \\
1681\end{array}$ & $\begin{array}{r}762 \\
1256 \\
1738\end{array}$ & $\begin{array}{r}705 \\
1410 \\
2115\end{array}$ \\
\hline
\end{tabular}

${ }^{\mathrm{a}}$ Ref. [36]; ${ }^{\mathrm{b}}$ Ref. [16, 24]; ${ }^{\mathrm{c}}$ Ref. [15]; ${ }^{\mathrm{d}}$ this work.

However large polyatomic colliders like heptane show a different picture if one compares the list of relaxing molecules. An increase of $\langle\Delta \mathrm{E}\rangle$ form 500 to $705 \mathrm{~cm}^{-1}(40 \%)$ at $\langle\mathrm{E}\rangle=10000 \mathrm{~cm}^{-1}$ corresponds to values between 766 and $1400 \mathrm{~cm}^{-1}(80 \%$ increase $)$ at $\langle\mathrm{E}\rangle=20000 \mathrm{~cm}^{-1}$ and 1116 to $2115 \mathrm{~cm}^{-1}$ (90\% increase) at $\langle\mathrm{E}\rangle=30000 \mathrm{~cm}^{-1}$ with a distributed, stepwise change between the extreme values on the table at each $\langle\mathrm{E}\rangle$. Within certain fluctuations $\mathrm{CO}_{2}$ seems to show a somewhat intermediate behaviour between the dependence found for large polyatomics and that for very small colliders; still with a rather large step in $\langle\Delta \mathrm{E}\rangle$ between azulene and biphenylene. Such interesting differences in variations of $\langle\Delta \mathrm{E}\rangle$ with the various larger relaxing energy donors can of course only be found with reasonable reliability if there are sufficiently consistent data sets available on certain members of "well behaved" molecules which overall represent standard properties without being a collection of conjectured "exceptions". To identify empirically extracted tendencies with related dynamical features on the basis of theoretical models is not a simple task because of the interplay of 
normally various coexisting tendencies. Detailed modelling on the basis of trajectory molecular dynamics calculations has become one of the most promising approaches in this direction.

Trajectory calculations with systematic studies on the role of various features of the interaction potential between collider and large target molecules have shown that only helium (possible also neon) is a clear cut case where the steepness of the repulsive wall is the domintating factor in collision efficiency with the well depth being rather secondary [27]. Thus one might rationalize the relative moderately increase in $\langle\Delta \mathrm{E}\rangle$ for biphenylene in Table 4 only for the example of $\mathrm{He}$ as indication of variations in the repulsive wall steepness in the potential. Already for argon - and thus for the larger rare gases - the potential well depth becomes the more important potential parameter with respect to the resulting $\langle\Delta E\rangle$, even if one takes into account the influences on the repulsive part which occur in some types of potential when the well depth is changed. One may again also refer to the favourable geometry of $\mathrm{Ar}-$ and $\mathrm{Kr}$ to a less extend - opposite to certain molecular ring structures as helpful for energy exchange. In the vast majority of trajectories "shattering" types of collisions are observed with very fast substantial forward-backward energy exchange on the fs-time scale within the "collision complex" before the final separation step occurs, usually involving only relatively moderate amounts of $\Delta \mathrm{E}$ [28]. With its two ring structures biphenylene can offer increased chances of favourable energy exchange kinematics.

The vibrational frequency spectrum of the relaxing molecule is clearly another important aspect of variations in $\langle\Delta \mathrm{E}\rangle$ in the same bath gas. With respect to the adiabaticity of a collision this idea had already become part of the early models, and in the extreme the lowest frequency alone has been considered as the "doorway" for the total vibrational relaxation. Recent trajectory calculations have clearly emphasized the strong influence of the lowest frequencies on $\langle\Delta \mathrm{E}\rangle$ of larger molecules, often with the lowest vibrational frequency as most important single parameter acting on $\langle\Delta \mathrm{E}\rangle$ [28]. Moreover it could be clearly demonstrated that also the character of such low frequency vibration is - understandibly - of much importance and that an out of plane bending in the energy donor [29] is much more efficient than some stretch vibration of the same frequency. Detailed inspection of the energy flow in all vibrations of azulene during molecular dynamics calculations of its relaxation have closer quantified these tendencies: Not only the lowest frequency, but about the five lowest frequency vibrations are responsible for about $80 \%$ of vibrationally released energy even in collisions with polyatomic molecules [30]. Table 6 gives a list of the lowest five vibrational frequencies of the molecules compared in Table 4 and their number of vibrations with frequencies less than $500 \mathrm{~cm}^{-1}$. The data show that biphenylene has a vibration with the very low frequency of $120 \mathrm{~cm}^{-1}$ and even a second one below the lowest frequency of all the other mol- 
Table 6. Lowest frequency vibrations of the molecules compared in Table 5.

\begin{tabular}{lll}
\hline & $\begin{array}{l}\text { Five lowest vibrational } \\
\text { frequencies } / \mathrm{cm}^{-1}\end{array}$ & $\begin{array}{l}\text { Number of vibrations } \\
\text { with } \tilde{v}<500 \mathrm{~cm}^{-1}\end{array}$ \\
\hline Pyridine & $373,406,601,652,703$ & 2 \\
Pyrazine & $350,418,602,704,756$ & 2 \\
Toluene & $217,347,406,467,524$ & 4 \\
Azulen & $189,240,304,323,331$ & 5 \\
Biphenylene & $120,175,339,366,395$ & 7 \\
\hline
\end{tabular}

ecules. A considerable relative increase of $\langle\Delta \mathrm{E}\rangle$ values in biphenylene can be expected along these lines. Finally the more evenly distributed increase of the $\langle\Delta \mathrm{E}\rangle$ in heptane in Table 6 from pyridine to biphenylene can be seen in accordance with expectations for increasingly dominant $\mathrm{V}-\mathrm{V}$ transfer which is also confirmed by trajectory results in case of large polyatomic colliders [28].

\subsection{Temperature dependence}

Our results on the temperature dependence of the measured relaxation rate constants $\mathrm{k}_{\mathrm{c}}(\mathrm{T})=(\mathrm{d}\langle\mathrm{E}(\mathrm{t})\rangle / \mathrm{dt}) \cdot\langle\Delta \mathrm{E}\rangle^{-1}$ are presented - following the usual convention in the field - as $\langle\Delta \mathrm{E}\rangle(\mathrm{T})=-\mathrm{k}_{\mathrm{c}}(\mathrm{T}) \cdot\left([\mathrm{M}] \cdot \mathrm{Z}_{\mathrm{LJ}}\right)^{-1} \cdot\langle\mathrm{E}\rangle$ with the standard Lennard-Jones collision number, $\mathrm{Z}_{\mathrm{LJ}}$, used for data reducation. (Problems arising with this choice of a temperature dependent normalization constant are discussed further down.) The numerical data in Table 3 and the examples for $\langle E\rangle=20000 \mathrm{~cm}^{-1}$ in Figs. 7-9 show very clearily that a negative temperature dependence in the range of $330-530 \mathrm{~K}$ is observed in all bath gases. This dependence is very weak in the rare gases, but generally increases with the complexity or efficiency of the collider to reach a decrease of about $40 \%$ within the $\Delta \mathrm{T}$ of $200 \mathrm{~K}$ in the case of heptane. These results are in good agreement with similar rather small negative temperature dependences found in recent direct relaxation measurements e.g. on azulene [4], toluene [5] and pyrazine [11].

However the literature also shows a choice of quite different results: Variation of the T-dependences in a range of $T^{ \pm n}(n \leq 1)$ has been reported for the deactivation of $\mathrm{CS}_{2}$ [31] and $\mathrm{SO}_{2}$ [32]. However for a triatomic molecule rather different behaviour from the typical one of large polyatomics may be expected as it is also found in the large variations of energy and collider dependences $\langle\Delta \mathrm{E}\rangle(\mathrm{E}, \mathrm{M})$ of triatomics. But also for large polyatomic molecules different results e.g. from practical no T-dependence (over wide ranges of temperature) [33] to strong positive dependences proportional to $\mathrm{T}$ [34] have been reported. The latter type of data are usually derived from very indirect sources, as e.g. from kinetic analysis of chemically or ther- 
mally activated reactive systems in the regime of pressure dependent kinetics. In view of the rather small temperature effects typically found by direct observation techniques special caution appears necessary with attempts to conclude on $\langle\Delta \mathrm{E}\rangle$ (or $\langle\Delta \mathrm{E}\rangle_{\text {down }}$ ) form experimental situations which would require at the same time perfect quantitative knowledge of e.g. reactive channels. Also severe misconceptions are possible apparently as consequence of too simplified models of energy transfer even when full master equation modelling is used in the fall-off regime. For instance the use of a constant $\alpha$ instead of linear energy dependent $\alpha(\mathrm{E})$ as minimum requirement in exponential transition probabilities $\mathrm{P}\left(\mathrm{E}^{\prime}, \mathrm{E}\right) \sim \exp \left[\left(\mathrm{E}^{\left.-\mathrm{E}^{\prime}\right) /}\right.\right.$ $\alpha(\mathrm{E})]$ used in a master equation can introduce misleading results on $\Delta \mathrm{E}(\mathrm{T})$ for rather large molecules at high temperatures. In such cases it can happen that part of the neglected internal energy dependence of $\Delta \mathrm{E}(\mathrm{E})$ (or $\alpha(\mathrm{E}))$ is projected into what then appears as a $\mathrm{T}$ dependence of $\Delta \mathrm{E}$, which will easily look positive linear dependent on $\mathrm{T}$.

From the point of view of a basic molecular collision theory it seems unavoidable to consider the relaxation rate constant $\mathrm{k}_{\mathrm{c}}=\mathrm{Z} \cdot \mathrm{P}\left(\mathrm{E}^{\prime}, \mathrm{E}\right)$ as a product of a collision number and a single collision energy transfer probability (for purely exponential relaxation this results in $\mathrm{k}_{\mathrm{c}}=-\mathrm{Z} \cdot[\mathrm{M}]\langle\Delta \mathrm{E}\rangle /$ $\langle E\rangle)$. It is however not easy to define the "correct" $Z(E, T)$. Already in connection with the energy dependence $\langle\Delta \mathrm{E}\rangle(\mathrm{E})$ the problem of a vibrational energy dependence $Z(E)$ is still unresolved and convincing methods to measure $Z(E)$ or $\langle\Delta E\rangle(E)$ separately do not yet exist. But in this case the standard approach of using an energy independent $\mathrm{Z}$ can be considered as a simple model or otherwise as some constant normalization factor without consequences for the results on e.g. the relative $\langle\Delta E\rangle(E)$ dependence. However such approach is not reasonable for the $\langle\Delta \mathrm{E}\rangle(\mathrm{T})$ dependence. In this case even the simplest physical model of $\mathrm{Z}$ cannot be constant but has to contain a temperature dependence, as e.g. the hardsphere model with $\mathrm{Z}_{\mathrm{HS}} \sim \mathrm{T}^{1 / 2}$. A "correct" splitting of the observed temperature dependence of $\mathrm{k}_{\mathrm{c}}$ on $\mathrm{Z}(\mathrm{T})$ and $\langle\Delta \mathrm{E}\rangle(\mathrm{T})$ is thus essential but not obvious. Some closer illustration shall be given in the following: In gas kinetics, when e.g. only the competition between energy transfer and reactive steps has to be considered one can always refer directly to $\mathrm{k}_{\mathrm{c}}$ and no problem with the T-dependence occurs. The corresponding values of $k_{c}$ are given in Table 4 . Between 333 and $523 \mathrm{~K}$ the relaxation rates slightly increase with temperature only in $\mathrm{He}$ $(10 \%)$ and $\mathrm{Ne}(7.5 \%)$. In all other bath gases they fall in this temperature range, between $6.5 \%$ (Ar) and $44 \%$ (heptane). Reduction with $\mathrm{Z}_{\mathrm{HS}}$ would reduce the values at $523 \mathrm{~K}$ by extra $25 \%$ more than at $333 \mathrm{~K}$. The temperature dependence of $Z_{\mathrm{LJ}}$ conventionally used for the rate data reduction shows collider specific T-dependencies due to variations in the collision integral $\Omega^{(2,2)^{*}}$ : For helium $\mathrm{Z}_{\mathrm{LJ}}$ increases from $333 \mathrm{~K}$ to $413 \mathrm{~K}$ by $6.8 \%$ and to $523 \mathrm{~K}$ by $15.5 \%$. This positive temperature dependence becomes weaker for the larger collider, is smallest for $\mathrm{CO}_{2}(0.1 \%$ at $413 \mathrm{~K}, 1.6 \%$ at $523 \mathrm{~K})$ and 
slightly negativ for the larger polyatomic colliders (heptane: $-0,6 \%$ $(413 \mathrm{~K})$ and $-0.7 \%(523 \mathrm{~K}))$. In our experiments $Z_{\mathrm{LJ}}$ is thus somewhat positively dependent on $\mathrm{T}$ for light rare gases but about independent on $\mathrm{T}$ for the larger molecular colliders. The results for $\langle\Delta \mathrm{E}\rangle /\langle\mathrm{E}\rangle$ from $\mathrm{k}_{\mathrm{c}} / \mathrm{Z}_{\mathrm{LJ}}$ are given in Table 4. They include some further convention: To make the numbers comparable $\sigma$ and $\varepsilon$ (Lennard-Jones) are taken from Ref. [19] as usually done in the field. However at least for the rare gases newer, better data from differential inelastic cross sections [25] are remarkably different with $\sigma 10 \%$ larger and $\varepsilon 6 \%(\mathrm{He})$ to $20 \%(\mathrm{Xe})$ larger. In addition the usual combination rules are shown to fail already for rare gas-rare gas combinations by 11 to $30 \%$. Such uncertainties may still be tolerable in the resulting effects on $\mathrm{Z}_{\mathrm{LJ}}$, however for the verification and modelling of the dynamics in trajectory calculations such uncertainties can be of crucial importance.

It is interesting to consider one extreme formal alternative in the problem of T-dependence, the assumption of temperature independent $\langle\Delta \mathrm{E}\rangle$ with all observed T-dependence then put into $Z(T)$. This can be done by assuming an attractive hardsphere potential (AHS) with a hardsphere repulsive wall and an attractive (exp-6) Lennard-Jones type potential for $\mathrm{r} \geq 2^{1 / 6} \cdot \sigma$. It has so far been used successfully for modelling $\langle\Delta \mathrm{E}\rangle(\varrho)$ dependences at very high fluid densities, when local density fluctuations become important, and for representation of $\langle\Delta \mathrm{E}\rangle(\mathrm{T})$ in azulene relaxation [4]. The rather weak and monotonous T-dependences of $k_{c}$ in this work can thus be represented within their error limits - but also with slight indications for systematic deviations - by the resulting $\mathrm{k}_{\mathrm{c}} \cdot \varrho \cdot \sqrt{\mathrm{T}} \exp \left(\varepsilon_{\mathrm{AHS}} / \mathrm{k}_{\mathrm{B}} \mathrm{T}\right)$, with $\varepsilon_{\mathrm{AHS}}$ used as an adjustable parameter to define an effective "AHS well depth". This is shown in Fig. 10. It is an advantage of this reduced data representation that it allows to present the $\langle\Delta \mathrm{E}\rangle(\mathrm{E}, \mathrm{T})$ dependences within compact expressions for each bath gas. However it has to be emphasized that such treatment is definitely not the physical solution of the T-dependence problem of $\langle\Delta \mathrm{E}\rangle$ and $\mathrm{Z}$. Consideration of single collisions shows immediatley that an assumed $\langle\Delta \mathrm{E}\rangle$ independent of $\mathrm{T}$ is at variance with experimental and trajectory results e.g. as it neglects any dependence of $\Delta \mathrm{E}$ on the kinetic energy even for a simple atomic collider.

A concentration on single collision events also emphasizes the basic equivalence of $\langle\Delta E\rangle(E)$ dependence on the vibrational energy of the "target" with the $\langle\Delta \mathrm{E}\rangle(\mathrm{T})$ dependence on the internal energy of the "collider" in a binary collision, which does not know the distinction between "collider" and "target" in the relevant centre of mass frame. In spite of this aspect of symmetry in two body collisions the resulting consequences in practical situations can appear very "unsymmetric": If e.g. hexafluorobenzene (HFB) is relaxed by a standard collider, the $\langle\Delta \mathrm{E}\rangle$ are very much larger than for benzene under equivalent conditions. However as a deactivating bath gas HFB behaves very much the same as comparable unfluorinated colliders [16]. 


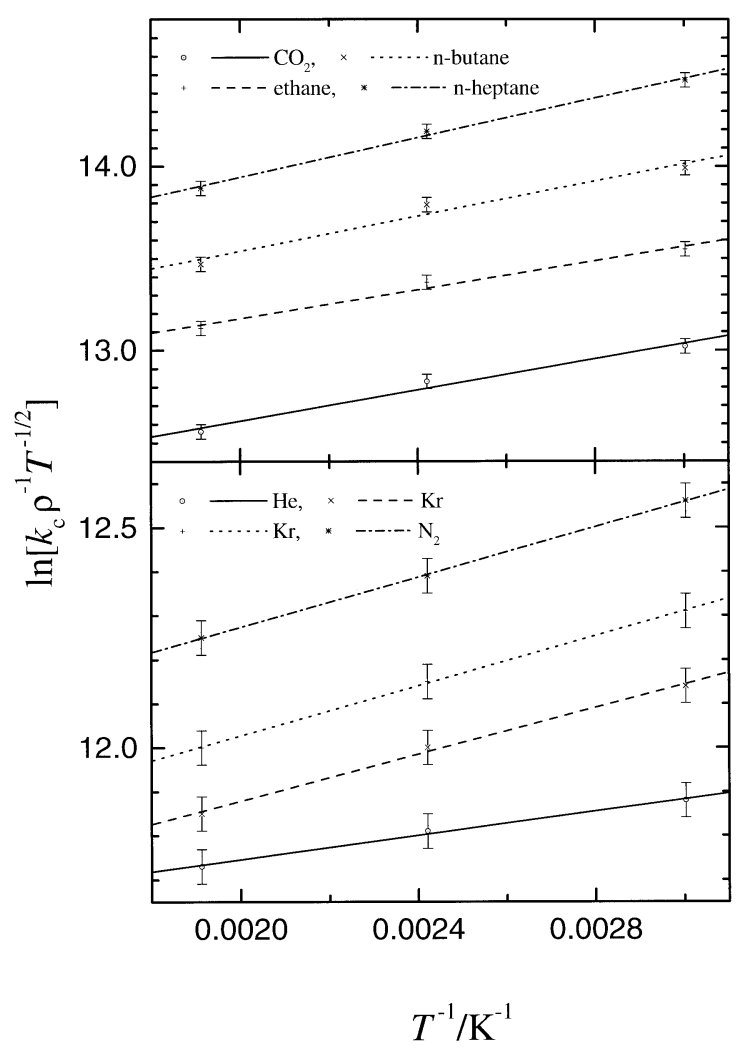

Fig. 10. Reduced representation of vibrational relaxation rates $k_{c}$ in various colliders as function of temperature. Within an "attractive hard sphere" model the slopes correspond to the effective well-depth $\varepsilon_{\mathrm{AHS}} \cdot \mathrm{k}_{\mathrm{B}}^{-1}$ of the biphenylene-collider interaction (see text).

Various basic dependences $\langle\Delta \mathrm{E}\rangle(\mathrm{T})$ are derived from fundamental models of collisional energy transfer. SSH-theory and its variants predict strong positive temperature dependence of V-T energy transfer, in connection with the strong influence of kinetic energy on energy exchange at a steep repulsive wall. Models of V-V transfer with dipol or similar attractive interaction in general result in negative T-dependences often with $\mathrm{T}^{-1}$. For the relaxation of diatomics very clear and instructive examples are known. $\mathrm{CO}$ relaxation in $\mathrm{CO}$ is very fast with an expected strong positive T-dependence. In contrast the NO-NO relaxation is orders of magnitude slower with only very weak, curved and partially negative T-dependence. Even this very different behaviour could fully be reconciled with model expectations by variation of the attractive well and a simple picture of a resulting trapping inner potential within centrifugal walls [35]. Indications of such general tenden- 
cies can also be recognized in the data of biphenylene relaxation. The negative temperature dependence which increases for larger polyatomic collider molecules is thus related to the increasing well depth of the interaction potential and the growing dominance of $\mathrm{V}-\mathrm{V}$ energy transfer for such colliders, as it is also strongly supported by related trajectory calculations [28].

Recent trajectory studies [36] on the effect of bath gas temperature on the relaxation of pyrazine have drawn a more detailed picture on the complex situation with large colliders in very good agreement with experimental data of negative temperature between 250 and $420 \mathrm{~K}$ for the relaxation of pyrazine in pyrazine [11]. The calculation show this clear negative Tdependence for a full range of various $\langle E\rangle$ at low temperatures. This dependence becomes weaker and then starts to rise slightly at higher temperatures. A qualitatively similar trend is found for argon as collider. In this case the rise of $\langle\Delta \mathrm{E}\rangle$ at higher temperatures is more pronounced and starts already at about $\mathrm{T}>200 \mathrm{~K}$ while some negative T-dependence is also found at $\mathrm{T}<200 \mathrm{~K}$. Some minimum in $\langle\Delta \mathrm{E}\rangle(\mathrm{E})$ occurs in both cases roughly in the vicinity of the respective values of $\varepsilon / \mathrm{k}_{\mathrm{B}}(222 \mathrm{~K}$ for Ar/pyrazine and $436 \mathrm{~K}$ for pyrazine/pyrazine). These results appear very promising for a dynamical interpretation with emphasize on the relative importance of collisional complex formation at the corresponding temperatures.

\section{Appendix}

The dependence of the average vibrational energy of biphenylene on temperature $\langle\mathrm{E}\rangle(\mathrm{T})$ was calculated according to $\langle\mathrm{E}\rangle=\mathrm{k}_{\mathrm{B}} \mathrm{T}^{2}\left(\delta \ln \mathrm{Z}_{\mathrm{vib}} / \delta \mathrm{T}\right)$ on the basis of the 54 frequencies given in Table 7. The result is shown in Fig. 11.

Table 7. Vibrational frequencies of biphenylene: (Experimental values from Ref. [37], calculated: *, from Ref. [38]).

\begin{tabular}{llllll}
\hline$\tilde{v}\left(\mathrm{~cm}^{-1}\right)$ & \multicolumn{7}{l}{} & \\
\hline 3074 & 1666 & 1288 & $1076^{*}$ & 765 & 567 \\
3072 & 1638 & 1267 & 1053 & 751 & $437 *$ \\
3072 & 1602 & 1260 & 1019 & $736^{*}$ & 395 \\
3057 & 1598 & $1194 *$ & $994^{*}$ & $735^{*}$ & $380^{*}$ \\
3057 & 1462 & 1166 & 989 & 733 & 366 \\
3030 & 1449 & 1151 & 975 & $655^{*}$ & $339 *$ \\
3022 & 1444 & 1128 & 962 & $628 *$ & 212 \\
3010 & 1426 & 1105 & $928 *$ & 612 & $175 *$ \\
$1840 *$ & 1399 & 1105 & 915 & 600 & 120 \\
\hline
\end{tabular}




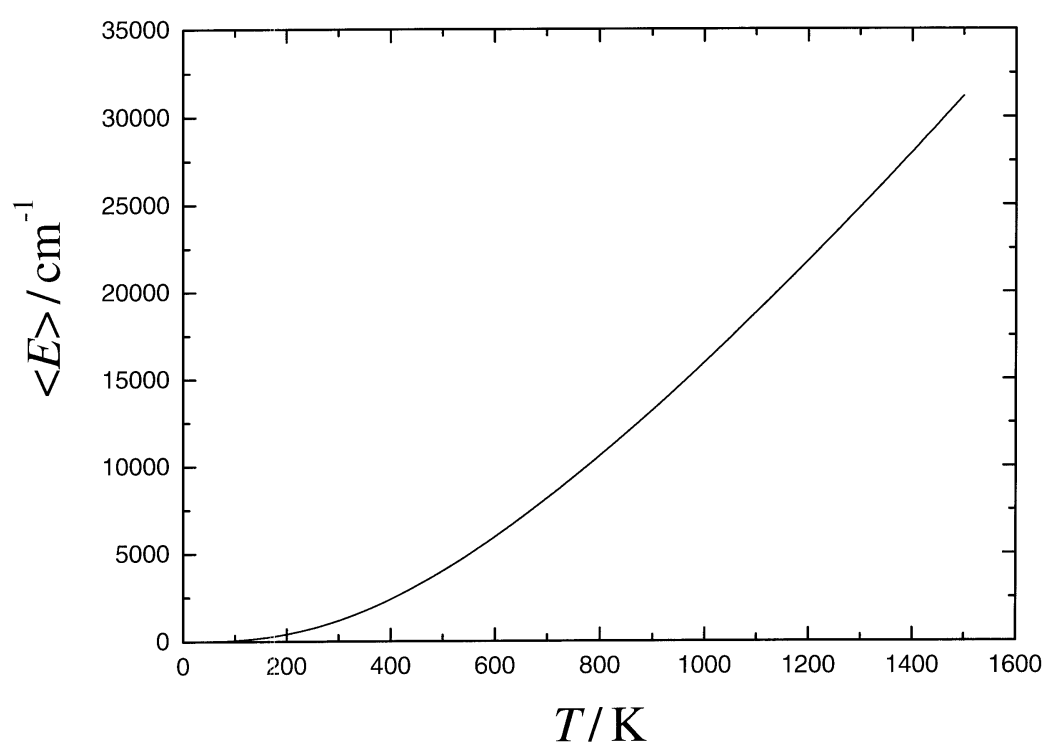

Fig. 11. Average vibrational energy $\langle E\rangle$ of biphenylene as function of temperature.

\section{Acknowledgements}

We thank Professor W. Lüttke for the generous sample of biphenylene, Dr. D. Schwarzer and Professor J. Troe for helpful discussions and the Deutsche Forschungsgemeinschaft, DFG (SFB 357 "Molekulare Mechanismen unimolekularer Prozesse") for financial support.

\section{References}

1. For reviews see e.g.: a) M. Quack and J. Troe, in "Gas Kinetics and Energy Transfer"; P. G. Ashmore and R. J. Donovan, Vol. 2, p. 175, London (1977); H. Hippler and J. Troe, in "Bimolecular Collisions", Eds. J. E. Baggott and M. N. R. Ashfold, p. 202, London (1989); b) D. C. Tardy and B. S. Rabinovitch, Chem. Rev. 77 (1977) 396; I. Oref and D. C. Tardy, Chem. Rev. 90 (1990) 1407; c) R. E. Weston Jr. and G. W. Flynn, Ann. Rev. Phys. Chem. 43 (1992) 559; G. W. Flynn, C. S. Parmenter and A. M. Wodtke, J. Phys. Chem. 100 (1996) 12817; d) Advances in Chemical Kinetics and Dynamics, Vol. 2, Ed. J. R. Barker, JAI Press, London (1995).

2. D. Schwarzer, J. Troe, M. Votsmeier and M. Zerezke, J. Chem. Phys. 105 (1996) 3121.

3. J. Benzler, S. Linkersdörfer and K. Luther, J. Chem. Phys. 106 (1997) 4992.

4. D. Schwarzer, J. Troe and M. Zerezke, J. Chem. Phys. 107 (1997) 8380.

5. H. Hippler, J. Troe and H. J. Wendelken, Chem. Phys. Lett. 84 (1981) 257; J. Chem. Phys. 78 (1983) 6709.

6. H. Hippler, K. Luther and J. Troe, J. Chem. Phys. 78 (1983) 6718; M. Damm, H. Hippler and J. Troe, ibid. 88 (1988) 3564.

7. M. Heymann, H. Hippler, D. Nahr, H. J. Plach and J. Troe, J. Phys. Chem. 92 (1988) 5507; J. E. Dove, H. Hippler, H. J. Plach and J. Troe, J. Chem. Phys. 81 (1984) 1209. 
8. H. Hippler, L. Lindemann and J. Troe, J. Chem. Phys. 83 (1985) 3906; H. Hippler, B. Otto and J. Troe, Ber. Bunsenges. Phys. Chem. 93 (1989) 428.

9. G. P. Smith and J. R. Barker, Chem. Phys. Lett. 78 (1981) 253; M. J. Rossi, J. R. Pladziewicz and J. R. Barker, J. Chem. Phys. 78 (1983) 6695

10. J. Shi and J. R. Barker, J. Chem. Phys. 88 (1988) 6219; B. M. Toselli and J. R. Barker, J. Chem. Phys. 95 (1991) 8108; J. R. Barker and B. M. Toselli, Int. Rev. Phys. Chem. 12 (1993) 305.

11. L. A. Miller and J. R. Barker, J. Chem. Phys. 105 (1996) 1383; L. A. Miller, C. D. Cook and J. R. Barker, J. Chem. Phys. 105 (1996) 3012.

12. G. V. Hartland, D. Qin and H.-L. Dai, J. Chem. Phys. 101 (1994) 8554; C. D. Pible, E. Sirota, J. Brenner and H.-L. Dai, J. Chem. Phys. 108 (1998) 1297.

13. W. Braun, M. D. Scheer and V. Kaufman, J. Res. Nat. Bur. Stand. 91 (1986) 313; K. M. Beck and R. J. Gordon, J. Chem. Phys. 87 (1987) 5681; B. M. Toselli, T. L. Walunas and J. R. Barker, J. Chem. Phys. 93 (1990) 4793.

14. H. G. Löhmannsröben and K. Luther, Chem. Phys. Lett. 144 (1988) 473; K. Luther and K. Reihs, Ber. Bunsenges. Phys. Chem. 92 (1988) 442; U. Hold, T. Lenzer, K. Luther, K. Reihs and A. Symonds, J. Chem. Phys. 112 (2000) 4076.

15. U. Hold, T. Lenzer, K. Luther, K. Reihs and A. Symonds, Ber. Bunsenges. Phys. Chem. 101 (1997) 552.

16. T. Lenzer, K. Luther, K. Reihs and A. Symonds, J. Chem. Phys. 112 (2000) 4090.

17. C. A. Michaels, A. S. Mullin, J. Park, J. Z. Chon and G. W. Flynn, J. Chem. Phys. 108 (1998) 2744; M. C. Wall, B. A. Stewart and A. S. Mullin, J. Chem. Phys. 108 (1998) 6185; M. C. Wall, A. E. Lernoff and A. S. Mullin, J. Chem. Phys. 111 (1999) 7373.

18. U. Griogleit, Diplom Thesis, Göttingen (1996).

19. J. O. Hirschfelder, C. F. Curtiss and R. B. Bird, Molecular Theory of Gases and Liquids, John Wiley, N. Y. C. (1954).

20. H. Hippler, J. Troe and H. J. Wendelken, Chem. Phys. Lett. 84 (1981) 257; L. Brouwer, H. Hippler, L. Lindemann and J. Troe, J. Phys. Chem. 89 (1985) 4608.

21. H. Hippler, J. Troe and H. J. Wendelken, J. Chem. Phys. 78 (1983) 5351; M. Quack, Nuovo Cimento B63 (1981) 1981.

22. P. Sulzer and K. Wieland, Helv. Phys. Acta 25 (1952) 653.

23. F. Deckert, Doctoral Thesis, Göttingen (1995).

24. U. Hold, U. Grigoleit, T. Lenzer and K. Luther, to be published.

25. G. Scoles, Ann. Rev. Phys. Chem. 31 (1980) 81.

26. P. D. Neufeld, A. R. Janzen and R. A. Aziz, J. Chem. Phys. 57 (1972) 1100.

27. T. Lenzer and K. Luther, J. Chem. Phys. 105 (1996) 10944.

28. T. Lenzer, K. Luther, J. Troe, R. G. Gilbert and K. F. Lim, J. Chem. Phys. 103 (1995) 626

29. G. Lendvay, J. Phys. Chem. 101 (1997) 9217.

30. V. S. Vikherenko, C. Heidelbach, D. Schwarzer, V. B. Nemtsov and J. Schroeder, J. Chem. Phys. 110 (1999) 5286.

31. J. E. Dove, H. Hippler and J. Troe, J. Chem. Phys. 82 (1985) 1907; M. Heymann, H. Hippler, H. J. Plach and J. Troe, J. Chem. Phys. 87 (1987) 3867.

32. M. Heymann, H. Hippler, D. Nahr, H. J. Plach and J. Troe, J. Phys. Chem. 92 (1988) 5507.

33. J. H. Kiefer and J. N. Shah, J. Phys. Chem. 91 (1987) 3024

34. Y. Feng, J. T. Niiranen, A. Bencsura, V. D. Knyazev and D. Gutman, J. Phys. Chem. 97 (1993) 871; M. A. Hanning-Lee, W. J. Green, M. J. Pilling, S. H. Robertson, J. Phys. Chem. 97 (1993) 860.

35. X. Yang and A. M. Wodtke, Int. Rev. Phys. Chem. 12 (1993) 123.

36. U. Grigoleit, Doctoral Thesis, Göttingen (1999).

37. A. Girlando and C. Pecile, J. Chem. Soc. Faraday Trans. 2, 69 (1973) 818.

38. J. C. Whitmer, S. J. Cyvin and B. N. Cyvin, Z. Naturforsch. 33a (1978) 45. 